\title{
On the spectrum of the sinh-Gordon model in finite volume
}

\author{
J. Teschner \\ DESY Theory, Notkestr. 85, 22603 Hamburg, Germany
}

Received 26 April 2007; accepted 22 January 2008

Available online 2 February 2008

\begin{abstract}
We derive a characterization of the spectrum of the sinh-Gordon model in terms of certain nonlinear integral equations. There exists a large class of solutions to these equations which allows a continuation between the infrared and the ultraviolet limits, respectively. We present nontrivial evidence for the claim that the class of solutions in question describes the spectrum of the sinh-Gordon model completely in both of these limits. The evidence includes some nontrivial relations to Liouville theory.
\end{abstract}

(c) 2008 Elsevier B.V. All rights reserved.

PACS: 03.70.+k; 11.10.Kk; 11.55.Ds; 02.60.Nm

Keywords: Integrable QFT; Sinh-Gordon model; Liouville

\section{Introduction}

We will discuss the quantization of the classical field theory which has the following Hamiltonian:

$$
H_{\mathrm{ShG}}=\int_{0}^{R} d x\left\{4 \pi \Pi^{2}+\frac{1}{16 \pi}\left(\partial_{\sigma} \varphi\right)^{2}+2 \mu \cosh (b \varphi)\right\} .
$$

Part of our motivation is the possible role of this model as a prototype for integrable models with non-compact target spaces like the nonlinear sigma models for non-compact symmetric spaces.

E-mail address: teschner@mail.desy.de. 
We will mainly consider the sinh-Gordon model in finite spacial volume which corresponds to imposing the periodic boundary conditions $\varphi(x+R)=\varphi(x)$. A lot is known about the sinhGordon model in infinite volume $R \rightarrow \infty$, see e.g. [1-5]. The spectrum contains a single particle with mass $m$ which is related to the parameter $\mu$ in (1.1) in a definite way [6]. The S-matrix factorizes into the product of two-particle scattering phase shifts $S\left(\vartheta_{1}-\vartheta_{2}\right)$, with

$$
S(\vartheta)=\frac{\sinh \vartheta-i \sin \vartheta_{0}}{\sinh \vartheta+i \sin \vartheta_{0}}, \quad \vartheta_{0} \equiv \frac{\pi b^{2}}{1+b^{2}}
$$

The known form-factors [2-4] completely characterize the corresponding local fields of the theory, at least in principle.

However, less is known about the case of finite $R$. Basically nothing is known about the spectrum of the model beyond certain characteristics of the ground state [7-9]. However, among these papers we would like to mention in particular [8] and [9] as important precursors of our investigation. The paper [8] discusses certain functional relations related to the thermodynamic Bethe ansatz equations which include the Baxter equation and the so-called quantum Wronskian relation. These functional relations will also play an important role in this paper. In [9] it was suggested that the separation of variables method (SOV-method) introduced by Sklyanin should be useful for this problem. Certain conjectures were proposed which would fully characterize the ground state expectation values of the fields $e^{\alpha \varphi}$. These conjectures were shown in [9] to pass highly nontrivial tests.

In order to get a solid groundwork for solving the sinh-Gordon model we have in joint work [10] with A. Bytsko introduced an integrable lattice regularization of the sinh-Gordon model. The construction of the Q-operator and the application of the SOV-method allowed us to characterize the spectrum of the model by identifying the precise conditions which ensure that a solution of the Baxter equation corresponds to a state in the spectrum.

In the present paper, which heavily builds upon [10] we will present nontrivial evidence in favor of the following claim. In order to formulate it, we will use the notation $\mathbf{t}=\left[\vartheta_{1}, \ldots, \vartheta_{M}\right]$, $\mathbf{k}=\left[k_{1}, \ldots, k_{M}\right]$ for vectors modulo permutations of their components. ${ }^{1}$

Claim 1. The Hilbert space $\mathcal{H}_{\mathrm{SG}}$ of the sinh-Gordon model contains a sector $\mathcal{H}_{\mathrm{TBA}}$ which exists for all $R>0$ and is complete within $\mathcal{H}_{\mathrm{SG}}$ both in the infrared (IR) limit $R \rightarrow \infty$ and the ultraviolet $(U V)$ limit $R \rightarrow 0$, respectively.

$\mathcal{H}_{\mathrm{TBA}}$ decomposes into subspaces $\mathcal{H}_{M}$ as $\mathcal{H}_{\mathrm{TBA}}=\bigoplus_{M=0}^{\infty} \mathcal{H}_{M}$. The sectors $\mathcal{H}_{M}$ have an orthonormal basis spanned by eigenvectors $e_{\mathbf{k}}$ to all the conserved quantities of the sinh-Gordon model which are labeled by tuples $\mathbf{k}=\left[k_{1}, \ldots, k_{M}\right] \in \mathbb{Z}^{M}$ of integers. The eigenvalue $E_{\mathbf{k}}$ of the Hamiltonian in the eigenstate $e_{\mathbf{k}}$ can then be expressed as

$$
E_{\mathbf{k}}=\sum_{a=1}^{M} m \cosh \vartheta_{a}-m \int_{\mathbb{R}} \frac{d \vartheta}{2 \pi} \cosh \vartheta \log \left(1+Y_{\mathbf{t}}(\vartheta)\right)
$$

where the function $Y_{\mathbf{t}}(\vartheta)$ and the tuple $\mathbf{t}=\left[\vartheta_{1}, \ldots, \vartheta_{M}\right]$ of real numbers are defined as follows.

\footnotetext{
1 This means that $\left[\ldots, k_{a}, \ldots, k_{b}, \ldots\right]=\left[\ldots, k_{b}, \ldots, k_{a}, \ldots\right]$ with $k_{a}=k_{b}$ being allowed.
} 
(i) For each tuple $\mathbf{t}$ of real numbers one may define $Y_{\mathbf{t}}(u)$ as the unique solution to the equations

$$
\begin{aligned}
& \text { (I) } \log Y_{\mathbf{t}}(\vartheta)-\int_{\mathbb{R}} \frac{d \vartheta^{\prime}}{2 \pi} \sigma\left(\vartheta-\vartheta^{\prime}\right) \log \left(1+Y_{\mathbf{t}}\left(\vartheta^{\prime}\right)\right) \\
& +m R \cosh \vartheta+\sum_{a=1}^{M} \log S\left(\vartheta-\vartheta_{a}-i \frac{\pi}{2}\right)=0,
\end{aligned}
$$

where the kernel $\sigma(\vartheta)$ is related to the function $S(\vartheta)$ via $\sigma(\vartheta) \equiv \frac{d}{d \vartheta} \arg S(\vartheta)$.

(ii) There is a unique solution $\mathbf{t}=\mathbf{t}(\mathbf{k} ; R)$ to the system of equations

$$
\begin{aligned}
& \text { (B) } m R \sinh \vartheta_{a}+\sum_{\substack{b=1 \\
b \neq a}}^{M} \arg S\left(\vartheta_{a}-\vartheta_{b}\right) \\
& \quad+i \int_{\mathbb{R}} \frac{d \vartheta}{2 \pi} \sigma\left(\vartheta_{a}-\vartheta+i \frac{\pi}{2}\right) \log \left(1+Y_{\mathbf{t}}(\vartheta)\right)=2 \pi k_{a},
\end{aligned}
$$

such that the leading asymptotics of the tuples $\mathbf{t}(\mathbf{k} ; R)$ for $R \rightarrow \infty$ is given by the tuples $\mathbf{t}_{0}(\mathbf{k})$ which are the unique solutions to the equations obtained from $(\mathrm{B})$ by setting $Y_{\mathbf{t}}(\vartheta) \equiv 0$.

In order to support our claim we will proceed as follows.

- In Section 2 we will rigorously derive the lattice counterparts $(\mathrm{I})_{\mathrm{L}}$ and $(\mathrm{B})_{\mathrm{L}}$ of Eqs. (I) and (B) from the results of [10].

- It is then observed in Section 3 that the continuum limit of these equations yields minor generalizations of Eqs. (I) and (B) above. We identify the conditions on the solutions of these equations which describe the spectrum of the sinh-Gordon model.

- In Section 4 we show that $\mathcal{H}_{\mathrm{TBA}}$ is complete in $\mathcal{H}_{\mathrm{SG}}$ in the infrared (IR) limit $R \rightarrow \infty$.

- Section 5 discusses the UV asymptotics $R \rightarrow 0$. Comparing the description of $\mathcal{H}_{\text {TBA }}$ given above to the predictions which follow from the expected relation with Liouville theory we find nontrivial evidence for the claim that $\mathcal{H}_{\mathrm{SG}} \simeq \mathcal{H}_{\mathrm{TBA}}$ in the $\mathrm{UV}$.

Given that $\mathcal{H}_{\mathrm{TBA}}$ appears to be complete in $\mathcal{H}_{\mathrm{SG}}$ both in the UV and the IR, it is tempting to speculate that the following conjecture might be true.

Conjecture 1. We have $\mathcal{H}_{\mathrm{SG}}=\mathcal{H}_{\mathrm{TBA}}$ for all values of $R$.

It is worth noting that the special case where the particle number $M$ is zero reproduces the thermodynamic Bethe ansatz (TBA) equation for the sinh-Gordon model [7]. Our proposal may therefore be seen as describing the generalization of the TBA to arbitrary excited states.

For $M \neq 0$ one may view Eqs. (B) as quantization conditions similar to the Bethe ansatz equations which determine the spectrum. The term in (B) which contains $Y_{\mathbf{t}}(\vartheta)$ represents finite volume corrections which are exponentially small when $R \rightarrow \infty$. The leading behavior for $R \rightarrow \infty$ is then precisely what one expects on the basis of the fact that the infinite volume S-matrix factorizes into two-particle scattering phases, see Section 4.3 for more discussion. 
Although the representation above is not fully explicit, it is efficient in the following sense. First note that it will certainly be possible to solve Eqs. (I) and (B) numerically with the help of a computer. Compared to any direct approach to the diagonalization of the Hamiltonian (without exploiting integrability) we have reduced the computational complexity enormously. Secondly, the system of equations (I), (B) allows us to extract a lot of important information like information about the asymptotic behavior in the infrared $m R \rightarrow \infty$ or ultraviolet $m R \rightarrow 0$, respectively.

We should also admit that the discussion of the UV asymptotics that we can offer in Section 5 is neither fully rigorous nor complete. However, some of our observations appear to be fairly nontrivial and therefore worth being mentioned. In particular, by using certain results from [11] we are able to recover the Liouville reflection amplitude directly from the sinh-Gordon model, and to give an alternative derivation of the important result from [6] concerning the exact relation between the scale parameters defined in terms of the UV and the IR behavior of the sinh-Gordon model, respectively.

\section{Lattice sinh-Gordon model}

We briefly recall from [10] the definition of the lattice sinh-Gordon model followed by the description of its spectrum that was found in this reference.

\subsection{Definition of the lattice sinh-Gordon model}

\subsubsection{Hilbert space $\mathcal{H}$}

The Hilbert space $\mathcal{H}$ is defined as

$$
\mathcal{H}=\left(L^{2}(\mathbb{R})\right)^{\mathrm{N}} \text {. }
$$

It is defined uniquely up to unitary equivalence as the irreducible representation of the canonical commutation relations

$$
\left[\Pi_{n}, \varphi_{n}\right]=\frac{1}{2 \pi i} \delta_{n, m}
$$

by self-adjoint operators $\varphi_{n}, \Pi_{m}, n, m=1, \ldots, \mathrm{N}$. The operators $\varphi_{n}$ and $\Pi_{n}$ represent the discretized sinh-Gordon field and its canonical conjugate momentum, respectively.

\subsubsection{Conserved charges}

Let us define the monodromy matrix $\mathrm{M}$ as

$$
\mathrm{M}(u) \equiv\left(\begin{array}{ll}
\mathrm{A}(u) & \mathrm{B}(u) \\
\mathrm{C}(u) & \mathrm{D}(u)
\end{array}\right) \equiv L_{\mathrm{N}}(u) \cdots \cdots L_{2}(u) \cdot L_{1}(u),
$$

where the Lax-operator $L_{n}(u)$ is chosen as

$$
L_{n}(u) \equiv \frac{1}{i}\left(\begin{array}{cc}
i e^{\pi b \Pi_{n}}\left(1+e^{-2 \pi b\left(\varphi_{n}+s\right)}\right) e^{\pi b \Pi_{n}} & e^{-\pi b s} \sinh \pi b\left(u+\varphi_{n}\right) \\
e^{-\pi b s} \sinh \pi b\left(u-\varphi_{n}\right) & i e^{-\pi b \Pi_{n}}\left(1+e^{2 \pi b\left(\varphi_{n}-s\right)}\right) e^{-\pi b \Pi_{n}}
\end{array}\right) .
$$

Define the operators $\mathrm{T}_{m}$ by the expansion

$$
\mathrm{T}(u)=\operatorname{tr}(\mathrm{M}(u))=e^{\pi b N u} \sum_{m=0}^{\mathrm{N}}\left(-e^{-2 \pi b u}\right)^{m} \mathrm{~T}_{m} .
$$

The operators $\mathrm{T}_{n}, n=1, \ldots, \mathrm{N}$, are independent, positive self-adjoint and mutually commuting. We will denote the set $\left\{T_{1}, \ldots, T_{N}\right\}$ of conserved charges by $\mathfrak{C}$. 


\subsubsection{Hamiltonian}

It was shown in [10] that there exists an operator $\mathrm{H}$ which has the following properties:

(i) $\mathrm{H}$ is self-adjoint.

(ii) $\mathrm{H}$ commutes with $\mathrm{T}_{n}, n=1, \ldots, \mathrm{N}$.

(iii) The classical continuum limit of $\mathrm{H}$ yields (1.1).

The explicit expression for $\mathrm{H}$ is neither simple nor important for the following. We take properties (i)-(iii) as justification for calling the quantum theory defined by the triple of objects

$$
(\mathcal{H}, \mathfrak{C}, \mathrm{H})
$$

the lattice sinh-Gordon model. The fact that the lattice sinh-Gordon model has equally many conserved charges as it has degrees of freedom suggests that one may approach the spectral problem for the Hamiltonian $H$ via the spectral problem for the conserved charges $\mathfrak{C}=\left\{T_{1}, \ldots, T_{N}\right\}$.

\subsection{Characterization of the spectrum}

\subsubsection{Quantization conditions}

The main result in [10] may be summarized as follows.

Claim 2. A function $t(u)$ is a joint eigenvalue of the family of operators $\mathrm{T}(u)$ if and only if it is $i b^{-1}$-periodic, $t(u)=t(u+i / b)$, and there exists a function $q_{t}(u)$ related to $t(u)$ by

$(\mathrm{Ba})_{\mathrm{L}} \quad t(u) q_{t}(u)=(a(u))^{\mathrm{N}} q_{t}(u-i b)+(d(u))^{\mathrm{N}} q_{t}(u+i b)$,

where $d(u)=a(-u)=1+e^{-2 \pi b\left(u+s+i \frac{b}{2}\right)}$, which has the following properties,

$\left(\mathrm{Q}_{1}\right)_{\mathrm{L}} q_{t}(u) \sim \exp \left(-i \frac{\pi}{2} \mathrm{~N}(u \mp s \mp i \delta)^{2}\right)$ for $|u| \rightarrow \infty,|\arg ( \pm u)|<\frac{\pi}{2}$,

$\left(\mathrm{Q}_{2}\right)_{\mathrm{L}} q_{t}(u)$ is meromorphic with poles of maximal order $\mathrm{N}$ in $\pm \Upsilon_{-s}$, the poles at $s \pm u=i \delta$ have order $\mathrm{N}$.

The set $\Upsilon_{s}$ is defined as $\Upsilon_{s}=s+i \delta+i b \mathbb{Z}^{\geqslant 0}+i b^{-1} \mathbb{Z}^{\geqslant 0}$ where $2 \delta \equiv b+b^{-1}$.

It will also be useful to observe that the relevant functions $q_{t}(u)$ can be characterized as solutions to a bilinear difference equation that only involves $q_{t}(u)$ itself.

Proposition 1. $q_{t}(u)$ has to satisfy the so-called quantum Wronskian condition,

$$
(\mathrm{Wr})_{\mathrm{L}} \quad q_{t}(u+i \delta) q_{t}(u-i \delta)-q_{t}\left(u+i \delta^{\prime}\right) q_{t}\left(u-i \delta^{\prime}\right)=W(u),
$$

where we have used the notations $\delta^{\prime} \equiv \delta-b$ and $W(u) \equiv\left[e^{i \pi\left(u^{2}+\sigma^{2}\right)} D_{\sigma}(u)\right]^{-\mathrm{N}}$, with $D_{\sigma}(u)$ being a special function whose definition and properties are recalled in Appendix $A$.

The fact that the functions $q_{t}(u)$ in the lattice sinh-Gordon model satisfy Eq. $(\mathrm{Wr})_{\mathrm{L}}$ was shown in [10]. There is also an argument of F. Smirnov [12] which may easily be adapted to deduce Eq. $(\mathrm{Wr})_{\mathrm{L}}$ directly from the conditions formulated in Claim 2. 


\subsubsection{SOV representation}

It was furthermore shown in [10] that $\mathcal{H}$ is unitarily equivalent to the space $\mathcal{H}_{\text {SOV }} \equiv$ $L^{2}\left(\mathbb{R}^{\mathrm{N}}, d \mu\right)^{\text {Symm }}$ which consists of functions $\Psi(\mathbf{y}), \mathbf{y}=\left(y_{1}, \ldots, y_{\mathrm{N}}\right)$, that are square-integrable w.r.t. the measure

$$
d \mu=\prod_{a=1}^{\mathrm{N}} d y_{a} \prod_{a>b} 4 \sinh \pi b\left(y_{a}-y_{b}\right) \sinh \pi b^{-1}\left(y_{a}-y_{b}\right),
$$

and totally symmetric under all permutations of the variables $y_{a}$. The representation $\mathcal{H}_{\mathrm{SOV}}$ is essentially characterized by the following two properties:

(i) The family of operators $\mathrm{B}(u)$ is diagonal,

$$
\mathrm{B}(u) \Psi(\mathbf{y})=b_{\mathbf{y}}(u) \Psi(\mathbf{y}),
$$

where the eigenvalue $b_{\mathbf{y}}(u)$ has been parametrized in terms of the variables $y_{a}$ as

$$
b_{\mathbf{y}}(u) \equiv-\prod_{a=1}^{\mathrm{N}} e^{-\pi b s} 2 i \sinh \pi b\left(u-y_{a}\right) .
$$

(ii) Eigenstates $\left|\Psi_{t}\right\rangle$ of $\mathrm{T}(u)$ with eigenvalue $t(u)$ are represented by the wave-function

$$
\Psi_{t}(\mathbf{y})=\prod_{a=1}^{\mathrm{N}} q_{t}\left(y_{a}\right)
$$

where $q_{t}(u)$ is an element of the set $\mathcal{Q}$ define above.

It is worth keeping in mind that $q_{t}(u)$ not only represents the eigenstates $\left|\Psi_{t}\right\rangle$ via (2.3), it also gives a generating function for all the conserved charges of the theory.

\subsection{Reformulation in terms of integral equations}

The next step will be to reformulate the conditions characterizing the spectrum in terms of nonlinear integral equations. The use of nonlinear integral equations as a reformulation of the functional relations that characterize the finite volume spectrum of integrable models goes back to [13].

It will be convenient to consider the functions $Q(\vartheta)$ defined by $Q(\vartheta) \equiv q(u)$ if $\vartheta=\frac{\pi}{2} \frac{u}{\delta}$. Abusing notation slightly we shall write $Q \in \mathcal{Q}$ if the function $q$ corresponding to $Q$ is contained in $\mathcal{Q}$. Let $\mathbb{S}$ be the strip $\mathbb{S} \equiv\left\{z \in \mathbb{C} ;|\operatorname{Im}(z)| \leqslant \frac{\pi}{2}\right\}$ and let $\partial \mathbb{S} \equiv\left\{z \in \mathbb{C} ;|\operatorname{Im}(z)|=\frac{\pi}{2}\right\}$.

We will then define $\mathcal{Q}_{M}$ to be the subset of $\mathcal{Q}$ which consists of functions $Q(\vartheta)$ for which

$\left(\mathrm{Q}_{3}\right)_{\mathrm{L}}$ there exists a subset $\mathbf{Z}=\left\{\vartheta_{1}, \ldots, \vartheta_{M}\right\} \subset \mathbb{S}$ such that $Q(\vartheta)$ satisfies $Q\left(\vartheta_{a}\right)=0$ for $a=1, \ldots, M$ and does not vanish elsewhere in $\mathbb{S}$.

If $\vartheta_{a}$ is a zero of $Q(\vartheta)$ of the order $m$ we will by convention assume that the exists a subset $\mathcal{I}_{a} \subset\{1, \ldots, M\}$ of cardinality $m$ such that $\vartheta_{a}=\vartheta_{b}$ if $b \in \mathcal{I}_{a}, \vartheta_{a} \neq \vartheta_{b}$ otherwise. It turns out that the parameters $\vartheta_{a}$ are particularly important characteristics of the elements $Q \in \mathcal{Q}$. 


\subsubsection{The integral equations}

Our aim will be to show that the elements of $\mathcal{Q}_{M}$ are in one-to-one correspondence to the elements of a certain set $\mathcal{Y}_{M}$ of solutions to the following nonlinear integral equation,

$$
\begin{aligned}
(\mathrm{I})_{\mathrm{L}} \quad \log Y(\vartheta)= & \int_{\mathcal{C}} \frac{d \vartheta^{\prime}}{4 \pi} \sigma\left(\vartheta-\vartheta^{\prime}\right) \log \left(W\left(\vartheta^{\prime}\right)+Y\left(\vartheta^{\prime}\right)\right) \\
& -\mathrm{N} \arctan \left(\frac{\cosh (\vartheta+i \tau)}{\sinh \sigma}\right)-\mathrm{N} \arctan \left(\frac{\cosh (\vartheta-i \tau)}{\sinh \sigma}\right) \\
& -\sum_{a=1}^{M^{\prime}} \log S\left(\vartheta-\vartheta_{a}-i \frac{\pi}{2}\right)-\frac{1}{2} \sum_{a=M^{\prime}+1}^{M} \log S\left(\vartheta-\vartheta_{a}-i \frac{\pi}{2}\right) .
\end{aligned}
$$

In order to formulate the integral equation $(\mathrm{I})_{\mathrm{L}}$ we have been using the following definitions:

- The following abbreviations appear in $(\mathrm{I})_{\mathrm{L}}$ :

$$
\sigma(\vartheta)=\frac{4 \sin \vartheta_{0} \cosh \vartheta}{\cosh 2 \vartheta-\cos 2 \vartheta_{0}}, \quad \vartheta_{0}=\frac{\pi}{2} \frac{b}{\delta}, \quad \sigma \equiv \frac{\pi}{2} \frac{s}{\delta}, \quad \tau \equiv \frac{\pi}{2} \frac{\delta^{\prime}}{\delta} .
$$

- We have assumed that the parameters $\vartheta_{a}$ satisfy $\vartheta_{a} \in \mathbb{S} \backslash \partial \mathbb{S}$ for $a=1, \ldots, M^{\prime}, \vartheta_{a} \in \partial \mathbb{S}$ for $a=M^{\prime}+1, \ldots, M$.

- The contour $\mathcal{C}$ in the integral equation $(\mathrm{I})_{\mathrm{L}}$ is defined by $\mathcal{C}=(\mathbb{R}+i 0) \cup(\mathbb{R}-i 0)$. The branch of the logarithm function in $(\mathrm{I})_{\mathrm{L}}$ is the principal value with branch cuts starting at the zeros and poles of $W\left(\vartheta^{\prime}\right)+Y\left(\vartheta^{\prime}\right)$ running to $-\infty$.

Definition 1. Let $\mathcal{Y}_{M}$ be the set of all solutions to the integral equation $(\mathrm{I})_{\mathrm{L}}$ such that

$\left(\mathrm{Y}_{1}\right)_{\mathrm{L}} \log Y(\vartheta) \sim-i \frac{2}{\pi} \delta^{2} \mathrm{~N}\left(\left(\vartheta \mp \sigma \mp i \frac{\pi}{2}\right)^{2}-\tau^{2}\right)$ for $|\vartheta| \rightarrow \infty,|\arg ( \pm \vartheta)|<\frac{\pi}{2}$,

$\left(\mathrm{Y}_{2}\right)_{\mathrm{L}} Y(\vartheta)$ is meromorphic with poles of maximal order $\mathrm{N}$ in $\pm \frac{\pi}{2 \delta}\left(\Upsilon_{-s+i \tau} \cup \Upsilon_{-s-i \tau}\right)$,

$\left(\mathrm{Y}_{3}\right)_{\mathrm{L}}$ We have $W(\vartheta)+Y(\vartheta)=0$ if $\vartheta=\vartheta_{a} \pm i \frac{\pi}{2}$ for $a=1, \ldots, M$.

Condition $\left(\mathrm{Y}_{3}\right)_{\mathrm{L}}$ implies severe restrictions on the parameters $\vartheta_{a}, a=1, \ldots, M$, which can be written as the set of equations

$$
\begin{gathered}
(\mathrm{B})_{\mathrm{L}} \quad \pi\left(2 k_{a}+1\right)-i \log W\left(\vartheta_{a}\right)-\int_{\mathcal{C}} \frac{d \vartheta}{4 \pi} \tau\left(\vartheta_{a}-\vartheta\right) \log (W(\vartheta)+Y(\vartheta)) \\
+i \mathrm{~N} \arctan \left(\frac{\sinh \left(\vartheta_{a}+i \tau\right)}{i \sinh \sigma}\right)+i \mathrm{~N} \arctan \left(\frac{\sinh \left(\vartheta_{a}-i \tau\right)}{i \sinh \sigma}\right) \\
=\sum_{b=1}^{M^{\prime}} \arg S\left(\vartheta_{a}-\vartheta_{b}\right)+\frac{1}{2} \sum_{b=M^{\prime}+1}^{M} \arg S\left(\vartheta_{a}-\vartheta_{b}\right),
\end{gathered}
$$

where

$$
\tau(\vartheta) \equiv-\frac{4 \sin \vartheta_{0} \sinh \vartheta}{\cosh 2 \vartheta+\cos 2 \vartheta_{0}}=-i \sigma\left(\vartheta+i \frac{\pi}{2}\right) .
$$




\subsubsection{The correspondence}

Our (first) main result is the following reformulation of the conditions which describe the spectrum of the lattice sinh-Gordon model:

Theorem 1. There is a one-to-one correspondence between the solutions $Y(\vartheta) \in \mathcal{Y}_{M}$ of the integral equations $(\mathrm{I})_{\mathrm{L}}$ and the elements $Q \in \mathcal{Q}_{M}$. This correspondence can be described as follows.

For a given element $Q \in \mathcal{Q}_{M}$ one gets the corresponding function $Y(\vartheta)$ via

$$
W(\vartheta)+Y(\vartheta)=Q\left(\vartheta+i \frac{\pi}{2}\right) Q\left(\vartheta-i \frac{\pi}{2}\right) .
$$

The set $\mathbf{Z}=\left\{\vartheta_{1}, \ldots, \vartheta_{M}\right\}$ is the set of zeros of $Q(\vartheta)$ within $\mathbb{S}$.

Conversely, given a solution $Y(\vartheta) \in \mathcal{Y}_{M}$ to $E q$. (I) $)_{\mathrm{L}}$ with $\vartheta_{a} \in \mathbb{S} \backslash \partial \mathbb{S}$ for $a=1, \ldots, M^{\prime}$ and $\vartheta_{a} \in \partial \mathbb{S}$ for $a=M^{\prime}+1, \ldots, M$, one defines the corresponding element $Q \in \mathcal{Q}_{M}$ as

$$
\begin{aligned}
(\mathrm{Q})_{\mathrm{L}} \quad \log Q(\vartheta)= & \int_{\mathcal{C}} \frac{d \vartheta^{\prime}}{4 \pi} \frac{\log \left(W\left(\vartheta^{\prime}\right)+Y\left(\vartheta^{\prime}\right)\right)}{\cosh \left(\vartheta-\vartheta^{\prime}\right)}-\mathrm{N} \arctan \left(\frac{\cosh \vartheta}{\sinh \sigma}\right) \\
& +\sum_{a=1}^{M^{\prime}} \int_{\mathcal{C}_{a}}^{\vartheta} d \vartheta^{\prime} \frac{1}{\sinh \left(\vartheta^{\prime}-\vartheta_{a}\right)}+\frac{1}{2} \sum_{a=M^{\prime}+1}^{M} \int_{\mathcal{C}_{a}}^{\vartheta} d \vartheta^{\prime} \frac{1}{\sinh \left(\vartheta^{\prime}-\vartheta_{a}\right)} .
\end{aligned}
$$

The functions $Q(u)$ defined by the formula $(\mathrm{Q})_{\mathrm{L}}$ are independent of the choice of contours $\mathcal{C}_{a}$ as long as they run from $-\infty$ to $\vartheta$, avoiding the singular points $\vartheta_{b}, b=1, \ldots, M$.

The proof of this theorem can be found in Appendix B.

\section{Continuum sinh-Gordon model}

\subsection{Continuum limit}

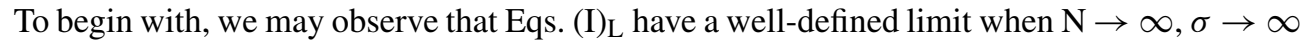
such that

$$
\frac{m R}{2 \sin \vartheta_{0}} \equiv 2 \mathrm{~N} e^{-\sigma}
$$

is kept constant. The limit may be carried out on the level of Eqs. $(\mathrm{I})_{\mathrm{L}}$ and leads to the system of equations

$$
\begin{aligned}
& \text { (I) } \log Y(\vartheta)+m R \cosh \vartheta-\int_{\mathcal{C}} \frac{d \vartheta^{\prime}}{2 \pi} \sigma\left(\vartheta-\vartheta^{\prime}\right) \log \left(1+Y\left(\vartheta^{\prime}\right)\right) \\
& -\sum_{a=1}^{M^{\prime}} \log S\left(\vartheta-\vartheta_{a}-i \frac{\pi}{2}\right)-\frac{1}{2} \sum_{a=M^{\prime}+1}^{M} \log S\left(\vartheta-\vartheta_{a}-i \frac{\pi}{2}\right)=0 .
\end{aligned}
$$

Definition 2. Let $\mathcal{Y}_{M}$ be the set of all solutions $Y(\vartheta)$ to the integral equation (I) such that $\left(\mathrm{Y}_{1}\right) Y(\vartheta)$ decays faster than exponentially for $|\operatorname{Re}(\vartheta)| \rightarrow \infty,|\operatorname{Im}(\vartheta)|<\frac{\pi}{2}$, 
$\left(\mathrm{Y}_{2}\right) Y(\vartheta)$ is entire analytic,

$\left(\mathrm{Y}_{3}\right)$ we have $1+Y(\vartheta)=0$ if $\vartheta=\vartheta_{a} \pm i \frac{\pi}{2}$ for $a=1, \ldots, M$.

The points $\vartheta_{a} \in \mathbb{S}$ are then restricted by the equations

$$
\begin{gathered}
\text { (B) } \pi\left(2 k_{a}-1\right)+m R \sinh \vartheta_{a}-\int_{\mathcal{C}} \frac{d \vartheta}{2 \pi} \tau\left(\vartheta_{a}-\vartheta\right) \log (1+Y(\vartheta)) \\
+\sum_{b=1}^{M^{\prime}} \arg S\left(\vartheta_{a}-\vartheta_{b}\right)+\frac{1}{2} \sum_{b=M^{\prime}+1}^{M} \arg S\left(\vartheta_{a}-\vartheta_{b}\right)=0,
\end{gathered}
$$

which is the same as what we obtain upon taking the limit (3.1) of the corresponding equations for the lattice sinh-Gordon model.

\subsection{Reformulation in terms of the Baxter equation}

Working backwards we may reformulate the previous description of the spectrum in terms of the Baxter equation. To each function $Y(\vartheta) \in \mathcal{Y}_{M}$ we can associate a function $Q(\vartheta)$ via

$$
\text { (Q) } \begin{aligned}
\log Q(\vartheta)= & -m R \frac{\cosh \vartheta}{2 \sin \vartheta_{0}}+\int_{\mathbb{R}} \frac{d \vartheta^{\prime}}{2 \pi} \frac{\log \left(1+Y\left(\vartheta^{\prime}\right)\right)}{\cosh \left(\vartheta-\vartheta^{\prime}\right)} \\
& +\sum_{a=1}^{M^{\prime}} \int_{\mathcal{C}_{a}}^{\vartheta} d \vartheta^{\prime} \frac{1}{\sinh \left(\vartheta^{\prime}-\vartheta_{a}\right)}+\frac{1}{2} \sum_{a=M^{\prime}+1}^{M} \int_{\mathcal{C}_{a}}^{\vartheta} d \vartheta^{\prime} \frac{1}{\sinh \left(\vartheta^{\prime}-\vartheta_{a}\right)} .
\end{aligned}
$$

We will regard the functions $q(u) \equiv Q\left(\frac{\pi}{2} \frac{u}{\delta}\right)$ that are defined in this way as the generating function for the conserved quantities of the continuum sinh-Gordon model.

Definition 3. Let $\mathcal{Q}$ be the set of all solutions $q(u)$ of the functional equation

(Wr) $q(u+i \delta) q(u-i \delta)-q\left(u+i \delta^{\prime}\right) q\left(u-i \delta^{\prime}\right)=1$,

which satisfy the conditions

$\left(\mathrm{Q}_{1}\right) q(u)$ is entire analytic,

$\left(\mathrm{Q}_{2}\right) \log q(u) \sim-\frac{m R}{2 \sin \vartheta_{0}} \cosh \frac{\pi}{2 \delta} u$ for $|\operatorname{Re}(u)| \rightarrow \infty,|\operatorname{Im}(u)|<\delta$.

Arguments very similar to those used in the proof of Theorem 1 then lead us to the following claim.

Claim 3. Eigenstates of the Hamiltonian of the continuum sinh-Gordon model are in one-to-one correspondence with the elements of $\mathcal{Q}$.

Let us note that $\mathcal{Q}$ is a certain set of solutions of Baxter's T-Q-relation

$$
t(u) q(u)=q(u+i b)+q(u-i b) .
$$

Indeed, given $q \in \mathcal{Q}$ let us use the notation $q_{+}(u) \equiv q(u), q_{-}(u) \equiv q(u-i / b)$ and define

$$
t(u) \equiv q_{+}(u+i b) q_{-}(u-i b)-q_{+}(u-i b) q_{-}(u+i b) .
$$


It is then easy to verify that $t$ and $q$ satisfy the T-Q-relation (3.2) with $t(u)$ being $i b^{-1}$-periodic, $t(u+i / b)=t(u)$.

\subsection{Discussion}

It seems reasonable to regard the conditions defining the set $\mathcal{Q}$ as an abstract characterization of the spectrum of the sinh-Gordon model. The zeros of $Q(\vartheta)$ contained in the strip $\mathbb{S}$ play a distinguished role.

In the following two sections we will present evidence that the class of solutions for which all the zeros of $Q(\vartheta)$ are located on the real line is of particular interest-this class of solutions will reproduce the particle picture in the IR and will allow us to make contact with Liouville theory in the UV. It seems likely that the states which correspond to this class of solutions are complete within $\mathcal{H}_{\mathrm{SG}}$.

It is therefore worth pointing out that the problem to classify the relevant solutions to Eqs. (I) and (B) for which $\vartheta_{a} \in \mathbb{R}$ for all $a=1, \ldots, M$ can be organized as follows. First note that we have

Proposition 2. There is a unique solution to Eq. (I) for any chosen tuple $\mathbf{t}=\left[\vartheta_{1}, \ldots, \vartheta_{M}\right]$ of real numbers.

Proof. The proof given in [14] for the case $M=0$ can easily be generalized to the case $M \neq 0$.

We may therefore define a tuple of functions $\Phi_{a}(\mathbf{t})$ by $\Phi_{a}(\mathbf{t})=\int_{\mathbb{R}} \frac{d \vartheta}{2 \pi} \tau\left(\vartheta_{a}-\vartheta\right) \log (1+$ $\left.Y_{\mathbf{t}}(\vartheta)\right), a=1, \ldots, M$, and regard the resulting equations $(\hat{\mathrm{B}})$,

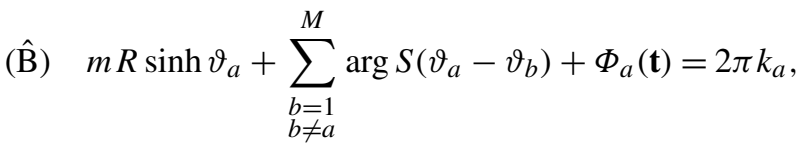

as a system of equations of Bethe ansatz type.

It will be shown in the next section that for any given tuple $\mathbf{k}=\left[k_{1}, \ldots, k_{M}\right]$ there exists a unique solution to $(\hat{\mathrm{B}})$ when $R$ is sufficiently large but still finite. It seems almost certain that this solution can be continued to all values of $R$ by means of Eqs. $(\hat{\mathrm{B}})$. Indeed, these equations can be used to derive equations which express $\frac{d}{d R} \vartheta_{a}$ in terms of certain real functions of $\mathbf{t}=$ $\left[\vartheta_{1}, \ldots, \vartheta_{M}\right]$. These equations do not exhibit singular behavior for any finite value of $R$, which makes us believe that the solutions found in the IR limit can be uniquely continued to arbitrary values of $R$. This prediction will be further supported by our discussion of the UV limit in Section 5. We therefore have little doubt that it is correct.

The solutions $\mathbf{t}(R)=\left[\vartheta_{1}(R), \ldots, \vartheta_{M}(R)\right]$ of Eqs. (B) that are defined in this way are uniquely determined by the tuples $\mathbf{k}=\left[k_{1}, \ldots, k_{M}\right]$ of integers which appear as data in Eqs. (B), i.e. $\mathbf{t}(R) \equiv \mathbf{t}(\mathbf{k} ; R)$. We will use the notation $Y_{\mathbf{k}}(\vartheta)$ and $Q_{\mathbf{k}}(\vartheta)$ for the corresponding $Y$ - and $Q$ functions, in particular $Y_{\mathbf{k}}(\vartheta) \equiv Y_{\mathbf{t}(\mathbf{k} ; R)}(\vartheta)$.

In order to arrive at our main claim formulated in the introduction it remains to accept the following conjecture.

Conjecture 2. The value $E_{\mathbf{k}}$ of the energy in the state $e_{\mathbf{k}}$ is given by formula (1.3). 
This conjecture is in particular supported by the observation that it reproduces the prediction for the ground state energy that is produced by the thermodynamic Bethe ansatz (TBA). The additional terms which appear in the case $M \neq 0$ represent the sum over the particle energies that one finds in the IR limit, cf. Section 4.3.

\section{The IR limit}

\subsection{Eq. (I)}

To begin with, let us make a few observation on the solutions to Eqs. (I) in the limit $R \rightarrow \infty$. First, let us observe that the asymptotics of the solutions $Y(\vartheta)$ to Eq. (I) for $|\vartheta| \rightarrow \infty$ is always determined by the "source term"

$$
\begin{array}{r}
J(\vartheta) \equiv \cosh \vartheta+\frac{1}{m R} \sum_{a=1}^{M^{\prime}} \log S\left(\vartheta-\vartheta_{a}-i \frac{\pi}{2}\right) \\
+\frac{1}{2 m R} \sum_{a=M^{\prime}+1}^{M} \log S\left(\vartheta-\vartheta_{a}-i \frac{\pi}{2}\right)
\end{array}
$$

as $Y(\vartheta) \sim e^{-m R J(\vartheta)}$. This is seen by noting that the kernel $\sigma\left(\vartheta-\vartheta^{\prime}\right)$ of the integral is peaked around $\vartheta=\vartheta^{\prime}$, decaying rapidly away from this region. This implies that the asymptotics of the integral in (I) for $|\vartheta| \rightarrow \infty$ is determined by the asymptotics of $Y(\vartheta)$. Taking into account the property $\left(\mathrm{Y}_{1}\right)$ allows us to conclude that the contribution of the integral in Eq. (I) becomes small when $|\vartheta| \rightarrow \infty$. This implies that $Y(\vartheta) \sim e^{-m R J(\vartheta)}$ for $|\vartheta| \rightarrow \infty$.

To continue it will be useful to regard Eq. (I) as a fixed point equation of the form $\mathrm{A} \cdot D=D$ for the deviation $D(\vartheta) \equiv \log Y(\vartheta)+m R J(\vartheta)$ of $\log Y(\vartheta)$ from its asymptotics $-m R J(\vartheta)$. The operator $A$ is the nonlinear integral operator defined by

$$
(\mathrm{A} \cdot D)(\vartheta) \equiv \int_{\mathbb{R}} \frac{d \vartheta^{\prime}}{2 \pi} \sigma\left(\vartheta-\vartheta^{\prime}\right) \log \left[1+e^{D\left(\vartheta^{\prime}\right)-m R J\left(\vartheta^{\prime}\right)}\right]
$$

We observe in particular that $\mathrm{A} \cdot D=D$ implies that $D$ is small of order $e^{-m R}$. We conclude that $Y$ is small of the order $e^{-m R}$ for $R \rightarrow \infty$ up to corrections which are suppressed by further factors of $e^{-m R}$.

\subsection{Eqs. (B)}

Turning our attention to Eqs. (B) we observe that $Y \sim e^{-m R}$ implies that the term which contains $Y$ will for large $m R$ be much smaller than the other terms. We therefore feel tempted to conclude that solutions to Eqs. (B) will be in one-to-one correspondence to the solutions of the equations which we obtain from (B) by dropping the term which contains $Y$. Let us therefore consider the following simplified system of equations:

$$
\pi\left(2 k_{a}-1\right)+m R \sinh \vartheta_{a}+\sum_{b=1}^{M^{\prime}} \arg S\left(\vartheta_{a}-\vartheta_{b}\right)+\frac{1}{2} \sum_{b=M^{\prime}+1}^{M} \arg S\left(\vartheta_{a}-\vartheta_{b}\right)=0 .
$$


It is sometimes also convenient to consider the exponentiated version of these equations

$$
e^{-2 i m R \sinh \vartheta_{a}}=-\prod_{a=1}^{M^{\prime}} S^{2}\left(\vartheta_{a}-\vartheta_{b}\right) \prod_{a=M^{\prime}+1}^{M} S\left(\vartheta_{a}-\vartheta_{b}\right) .
$$

Lemma 1. The solutions $\vartheta_{a}, a=1, \ldots, M$, are real.

Proof. We follow the idea from [15]. Note that

$$
|S(\vartheta)|^{2}=\frac{|\sinh \vartheta|^{2}+\left(\sin \vartheta_{0}\right)^{2}-2 \sin \vartheta_{0} \cosh (\operatorname{Re}(\vartheta)) \sin (\operatorname{Im}(\vartheta))}{|\sinh \vartheta|^{2}+\left(\sin \vartheta_{0}\right)^{2}+2 \sin \vartheta_{0} \cosh (\operatorname{Re}(\vartheta)) \sin (\operatorname{Im}(\vartheta))}
$$

from which it is easily read off that

$$
\left\{\begin{array}{l}
|S(\vartheta)| \leqslant 1 \\
|S(\vartheta)| \geqslant 1
\end{array}\right\} \quad \text { if } \quad\left\{\begin{array}{l}
0 \leqslant \operatorname{Im}(\vartheta) \leqslant \pi \\
-\pi \leqslant \operatorname{Im}(\vartheta) \leqslant 0
\end{array}\right\} .
$$

Let $\vartheta_{\max }$ be the component of $\mathbf{t}=\left[\vartheta_{1}, \ldots, \vartheta_{M}\right]$ which has maximal imaginary part. By taking the absolute value of both sides of (4.4) it follows that $\operatorname{Im}\left(\vartheta_{\max }\right) \leqslant 0$. Let $\vartheta_{\min }$ be the component of $\mathbf{t}=\left[\vartheta_{1}, \ldots, \vartheta_{M}\right]$ which has minimal imaginary part. In a way that is similar to the previous argument we infer that $\operatorname{Im}\left(\vartheta_{\min }\right) \geqslant 0$. Taken together we have $0 \leqslant \operatorname{Im}\left(\vartheta_{\min }\right) \leqslant \operatorname{Im}\left(\vartheta_{a}\right) \leqslant$ $\operatorname{Im}\left(\vartheta_{\max }\right) \leqslant 0$, from which our claim follows.

The lemma implies in particular that $M=M^{\prime}$, i.e. there are no components $\vartheta_{a}$ in $\mathbf{t}=$ $\left[\vartheta_{1}, \ldots, \vartheta_{M}\right]$ with $\vartheta_{a} \in \partial \mathbb{S}$. We will therefore now consider the equations

$$
\pi\left(2 k_{a}-1\right)+m R \sinh \vartheta_{a}+\sum_{b=1}^{M} \arg S\left(\vartheta_{a}-\vartheta_{b}\right)=0 .
$$

Lemma 2. For each choice of integers $\mathbf{k}=\left[k_{1}, \ldots, k_{M}\right]$ there is a unique solution $\mathbf{t}=$ $\left[\vartheta_{1}, \ldots, \vartheta_{M}\right]$ to Eqs. (4.7).

Proof. We follow the idea from [16]. Let us define

$$
P(\vartheta) \equiv \int_{0}^{\vartheta} d \vartheta^{\prime} \arg S\left(\vartheta^{\prime}\right)
$$

Eqs. (4.7) are then easily seen to be conditions for a local extremum of the function

$$
R(\mathbf{t}) \equiv \sum_{a=1}^{M}\left(m R \cosh \vartheta_{a}+2 \pi k_{a} \vartheta_{a}\right)+\sum_{a<b} P\left(\vartheta_{a}-\vartheta_{b}\right) .
$$

The matrix of second derivatives is positive definite,

$$
\sum_{a, b} v_{a} \frac{\partial^{2} R}{\partial \vartheta_{a} \partial \vartheta_{b}} v_{b}=\sum_{a=1}^{M} v_{a}^{2} m R \cosh \vartheta_{a}+\sum_{a<b}\left(v_{a}-v_{b}\right)^{2} \sigma\left(\vartheta_{a}-\vartheta_{b}\right)>0
$$

It follows that the function $R(\mathbf{t})$ has a unique minimum, which is the desired solution of (4.7). 
We would finally like to argue that existence and uniqueness of the solutions to Eqs. $(\hat{\mathrm{B}})$ will still hold if $R$ is sufficiently large but finite. Indeed, Eqs. $(\hat{\mathrm{B}})$ are the conditions for an extremum of the function

$$
\begin{aligned}
\hat{R}(\mathbf{t}) \equiv & \sum_{a=1}^{M}\left(m R \cosh \vartheta_{a}+2 \pi k_{a} \vartheta_{a}\right)+\sum_{a<b} P\left(\vartheta_{a}-\vartheta_{b}\right)+\int_{\mathbb{R}} \frac{d \vartheta}{2 \pi} F\left(Y_{\mathbf{t}}(\vartheta)\right) \\
& -\int_{\mathbb{R}} \frac{d \vartheta}{2 \pi} \int_{\mathbb{R}} \frac{d \vartheta^{\prime}}{2 \pi} \log \left(1+Y_{\mathbf{t}}(\vartheta)\right) \sigma\left(\vartheta-\vartheta^{\prime}\right) \log \left(1+Y_{\mathbf{t}}\left(\vartheta^{\prime}\right)\right),
\end{aligned}
$$

where $F(y) \equiv \int_{0}^{y} \frac{d t}{t} \log (1+t)$. The additional terms in (4.11) will be suppressed by factors of $e^{-m R}$. For large enough values of $R$ this is enough to conclude that their presence will not spoil positivity of the matrix of second derivatives, which leads us to the conclusion that existence and uniqueness of the solutions to $(\hat{\mathrm{B}})$ is ensured if $R$ is sufficiently large but still finite.

Remark 1. In similar problems it is often assumed that $k_{a} \neq k_{b}$ if $a \neq b$. It should be stressed that this assumption is not coming from the existence of solutions to Eqs. (4.7). Instead it is coming from additional requirements like the existence of wave-functions of a certain form associated to the solutions of equations like (4.7). In our case we have shown that all functions $Y(\vartheta) \in \mathcal{Y}_{M}$ correspond to states in the spectrum, and we furthermore argued that for large enough $R$ there exist functions $Y_{\mathbf{k}}(\vartheta) \in \mathcal{Y}_{M}$ corresponding to all tuples $\mathbf{k}=\left[k_{1}, \ldots, k_{M}\right]$, including those which may have $k_{a}=k_{b}$ for $a \neq b$.

\subsection{Relation to the particle picture}

We have seen that to leading order in $e^{-m R}$ one gets the spectrum from the solutions of Eqs. (4.7) of Bethe ansatz type. This can be understood more intuitively as follows. The interactions between a pair of particles in a massive relativistic quantum field theory like the sinh-Gordon model are expected to be relevant mainly if the mutual distance is of the order $1 / \mathrm{m}$, with rapid decay when the distance increases further. For small density $M / R$ it should therefore give a reasonable approximation to assume that the motion of the particles is approximately free for most of the time, except if two particles cross each other. In this case one should be able to describe the crossing of two particles in terms of the two-particle S-matrix. In order to get an approximate description for a state $\Psi_{\mathbf{t}}$ characterized by the rapidities $\mathbf{t}=\left[\vartheta_{1}, \ldots, \vartheta_{M}\right]$ one may try to represent it in terms of a coordinate space wave-functions $\Psi_{\mathbf{t}}(\mathbf{x}), \mathbf{x}=\left(x_{1}, \ldots, x_{M}\right)$. The idea that the motion is free except for two-particle crossings represented by the scattering phase shift $S\left(\vartheta_{a}-\vartheta_{b}\right)$ leads to the following ansatz for the wave-function $\Psi_{\mathbf{t}}(\mathbf{x})$,

$$
\Psi_{\mathbf{t}}(\mathbf{x})=\exp \left(i \sum_{a=1}^{M} p_{a} x_{a}\right) \sum_{p \in S_{M}} K_{p}(\mathbf{t}) \chi_{p}(\mathbf{x}),
$$

where $S_{M}$ is the set of all permutations $p$ of the elements of $\{1, \ldots, M\}, \chi_{p}(\mathbf{x})=1$ if $x_{p(1)}<$ $x_{p(2)}<\cdots<x_{p(M)}$ and zero otherwise, and $K_{p}(\mathbf{t})$ is defined up to a multiplicative constant by the property that the element $(a b) \in S_{M}$ which generates an exchange of the elements $a$ and $b$ of $\{1, \ldots, M\}$ is realized as

$$
K_{(a b) p}(\mathbf{t})=S\left(\vartheta_{a}-\vartheta_{b}\right) K_{p}(\mathbf{t}) .
$$


Requiring that the wave-function is periodic w.r.t. the particle coordinates $x_{a}$ when $x_{a} \rightarrow x_{a}+R$ then leads to the quantization conditions

$$
e^{i R p_{a}} \prod_{\substack{b=1 \\ b \neq a}}^{M} S\left(\vartheta_{a}-\vartheta_{b}\right)=1 .
$$

We see that our Bethe ansatz equations (B) describe finite volume deviations of order $e^{-m R}$ from the asymptotic Bethe ansatz equations (4.13).

\section{The ultraviolet limit}

We will now see that our proposal for the spectrum of the sinh-Gordon model seems to lead to a rather nice picture of its UV limit. Some qualitative and quantitative aspects of this picture will be found to be in remarkable agreement with certain predictions that follow from the expected relationship with Liouville theory [17].

\subsection{Partial decoupling of left- and right-movers}

\subsubsection{Clustering of the roots of $(B)$}

We would like to argue that the solutions $\mathbf{t}=\left[\vartheta_{1}, \ldots, \vartheta_{M}\right]$ of Eqs. (I) and (B) have the following behavior for $R \rightarrow 0$,

$$
\vartheta_{a}=\operatorname{sgn}\left(k_{a}\right)|\log m R|+\mathcal{O}(1) .
$$

This means that the particles with $\operatorname{sgn}\left(k_{a}\right)>0$ or $\operatorname{sgn}\left(k_{a}\right)<0$ are moving to the right or to the left with velocity close to the speed of light, respectively. The separations $\left|\vartheta_{a}-\vartheta_{b}\right|$ within each cluster are of the order one in comparison with $|\log m R|$.

In order to see this, let us rewrite Eqs. (I), (B) in terms of the variables $\theta_{a}$ and the functions $\tilde{Y}_{\mathbf{k}}^{ \pm}(\theta)$ which are defined respectively by

$$
\vartheta_{a}=\theta_{a}+\operatorname{sgn}\left(k_{a}\right)|\log m R|, \quad \tilde{Y}_{\mathbf{k}}^{ \pm}(\theta)=Y_{\mathbf{k}}(\theta \pm|\log m R|) .
$$

Let $\mathbb{K}_{ \pm}$be the sets of indices $a$ for which $\pm k_{a} \geqslant 0$ and let $\mathbb{K}_{0}$ be the set of indices $a$ for which $k_{a}=0$. Eqs. (I), (B) can then be written in the following form:

$$
\begin{gathered}
\left(\mathrm{I}_{ \pm}^{\prime}\right) \quad \log \tilde{Y}_{\mathbf{k}}^{ \pm}(\theta)-\int_{\mathbb{R}} \frac{d \theta^{\prime}}{2 \pi} \sigma\left(\theta-\theta^{\prime}\right) \log \left(1+\tilde{Y}_{\mathbf{k}}^{ \pm}\left(\theta^{\prime}\right)\right) \\
+\frac{1}{2} e^{ \pm \theta}+\sum_{a \in \mathbb{K}_{ \pm} \cup \mathbb{K}_{0}} \log S\left(\theta-\theta_{a}-i \frac{\pi}{2}\right)+\mathcal{O}(m R)=0, \\
\left(\mathrm{~B}_{ \pm}^{\prime}\right) \quad \pi\left(2 k_{a}-1\right)-\int_{\mathbb{R}} \frac{d \theta}{2 \pi} \tau\left(\theta_{a}-\theta\right) \log \left(1+\tilde{Y}_{\mathbf{k}}^{ \pm}(\theta)\right) \\
\pm \frac{1}{2} e^{ \pm \theta_{a}}+\sum_{b \in \mathbb{K}_{ \pm} \cup \mathbb{K}_{0}} \arg S\left(\theta_{a}-\theta_{b}\right)+\mathcal{O}(m R)=0 .
\end{gathered}
$$

We observe that the variables $\theta_{a}$ and the functions $\tilde{Y}_{\mathbf{k}}^{ \pm}(\theta)$ are constrained by equations which up to corrections of order $m R$ do not depend on the scale parameter $m R$ at all. The leading 
asymptotics of the functions $\tilde{Y}_{\mathbf{k}}^{ \pm}(\theta)$ will therefore be found within the set of solutions to the equations obtained from $\left(\mathrm{I}_{ \pm}^{\prime}\right),\left(\mathrm{B}_{ \pm}^{\prime}\right)$ by dropping all $m R$-dependent terms.

\subsubsection{The decoupled theories for left- and right-movers}

Let us consider the equations obtained from Eqs. $\left(\mathrm{I}_{ \pm}^{\prime}\right),\left(\mathrm{B}_{ \pm}^{\prime}\right)$ above by dropping the terms of order $\mathcal{O}(m R)$ and by undoing the shifts (5.2) in order to re-introduce a convenient $m R$ dependence. For reasons that will become clear later we will also temporarily drop the terms from the summations over $a \in \mathbb{K}_{\mathrm{o}}$ and consider the equations

$$
\begin{gathered}
\left(\mathrm{I}_{ \pm}\right) \quad \log Y^{ \pm}(\vartheta)-\int_{\mathbb{R}} \frac{d \vartheta^{\prime}}{2 \pi} \sigma\left(\vartheta-\vartheta^{\prime}\right) \log \left(1+Y^{ \pm}\left(\vartheta^{\prime}\right)\right) \\
+\frac{m R}{2} e^{ \pm \vartheta}+\sum_{a \in \mathbb{K}_{ \pm}} \log S\left(\vartheta-\vartheta_{a}-i \frac{\pi}{2}\right)=0, \\
\left(\mathrm{~B}_{ \pm}\right) \quad \pi\left( \pm 2 k_{a}-1\right)-\int_{\mathbb{R}} \frac{d \vartheta}{2 \pi} \tau\left(\vartheta_{a}-\vartheta\right) \log \left(1+Y^{ \pm}(\vartheta)\right) \\
\pm \frac{m R}{2} e^{ \pm \vartheta_{a}}+\sum_{b \in \mathbb{K}_{ \pm}} \arg S\left(\vartheta_{a}-\vartheta_{b}\right)=0 .
\end{gathered}
$$

The corresponding $Q$-functions are defined respectively by

$$
\begin{aligned}
\left(\mathrm{Q}_{ \pm}\right) \log Q^{ \pm}(\vartheta)= & -\frac{m R}{4 \sin \vartheta_{0}} e^{ \pm \vartheta}+\int_{\mathbb{R}} \frac{d \vartheta^{\prime}}{2 \pi} \frac{\log \left(1+Y_{\mathbf{k}}^{ \pm}\left(\vartheta^{\prime}\right)\right)}{\cosh \left(\vartheta-\vartheta^{\prime}\right)} \\
& +\sum_{a \in \mathbb{K}_{ \pm}} \int_{\mathcal{C}_{a}}^{\vartheta} d \vartheta^{\prime} \frac{1}{\sinh \left(\vartheta^{\prime}-\vartheta_{a}\right)}
\end{aligned}
$$

In a companion paper [18] we will show that these equations characterize the spectrum of a quantum integrable model that might be called the $c>1$ quantum $\mathrm{KdV}$ theory ${ }^{2}$ in order to distinguish it from its $c<1$ counterpart studied in [19].

It is very important to notice that the system of Eqs. $\left(\mathrm{I}_{ \pm}\right),\left(\mathrm{B}_{ \pm}\right)$has families of solutions that depend on an additional complex parameter $P$. This is a new feature compared to the case of Eqs. (I), (B). More precisely we have the following claim for which strong support will be given in [18].

Claim 4. (See [18].) For given tuples $\mathbf{k}=\left[k_{1}, \ldots, k_{M_{ \pm}}\right]$of integers with $k_{a}>0$ and each imaginary value $P \in i \mathbb{R}$ there exist solutions $\left(Y_{\mathbf{k}, P}^{ \pm}(\vartheta), \mathbf{t}_{\mathbf{k}, P}^{ \pm}\right)$of Eqs. $\left(\mathrm{I}_{ \pm}\right),\left(\mathrm{B}_{ \pm}\right)$, respectively, uniquely specified by the property that the corresponding functions $Q_{\mathbf{k}, P}^{ \pm}(\vartheta)$ have leading asymptotics for $\vartheta \rightarrow \mp \infty$ of the following form

$$
Q_{\mathbf{k}, P}^{ \pm}(\vartheta) \underset{\vartheta \rightarrow \mp \infty}{\sim} \frac{\cos (4 \delta P \vartheta \pm \Theta(\mathbf{k} \mid P))}{\sqrt{\sinh (2 \pi b P) \sinh \left(2 \pi b^{-1} P\right)}} .
$$

\footnotetext{
2 So far we had been assuming $b \in \mathbb{R}$ which would correspond to $c>25$. The existence of an analytic continuation to $c>1$ is nontrivial. It ultimately follows from the fact [23] that Liouville theory and, relatedly, the theory of representations of the Virasoro algebra have nice analytic properties w.r.t. to the value of $c$.
} 
The function $\Theta(\mathbf{k} \mid P)$, regarded as a function of $P$, are entire analytic and satisfy

(a) $\Theta(\mathbf{k} \mid P)$ is strictly monotonous.

(b) $\Theta(\mathbf{k} \mid P)=-\Theta(\mathbf{k} \mid-P)$.

The corrections to the asymptotics (5.3) are suppressed by (non-integral) powers of $m R e^{ \pm \vartheta}$.

It follows from the last statement in this claim that formula (5.3) may be expected to yield reasonable approximations for the functions $Q_{\mathbf{k}, P}^{ \pm}(\vartheta)$ as long as $\pm \vartheta>-|\log m R|+\Lambda$ if $\Lambda \ll$ $|\log m R|$ is such that $e^{-\Lambda} \ll 1$. When $m R$ is small one therefore expects to find a region

$$
\mathcal{P} \equiv\{\vartheta \in \mathbb{R} ;|\vartheta|<|\log m R|-\Lambda\},
$$

in which Eq. (5.3) provides good approximations for both $Q_{\mathbf{k}, P}^{+}(\vartheta)$ and $Q_{\mathbf{k}, P}^{-}(\vartheta)$, respectively. The region $\mathcal{P}$ defined in (5.4) will henceforth be called the plateau.

We will in the following be interested in cases where $P \in \mathbb{R}$ and $P=\mathcal{O}\left(|\log (m R)|^{-1}\right)$. Note that $Q_{\mathbf{k}, P}^{ \pm}(\vartheta)$ will then have infinitely many additional zeros within the strip $\mathbb{S}$, as indicated by the asymptotics (5.3). ${ }^{3}$ The corresponding functions $Y_{\mathbf{k}, P}^{ \pm}(\vartheta)$ will satisfy equations similar to $\left(\mathrm{I}_{ \pm}\right),\left(\mathrm{B}_{ \pm}\right)$which contain extra terms corresponding to the additional zeros of $Q_{\mathbf{k}, P}^{ \pm}(\vartheta)$. These extra zeros are precisely what is needed for describing the asymptotics of the solutions to ( $\mathrm{I}_{ \pm}^{\prime}$ ), $\left(\mathrm{B}_{ \pm}^{\prime}\right)$ in terms of the functions $Y_{\mathbf{k}, P}^{ \pm}(\vartheta)$ introduced in Claim 4.

\subsubsection{Residual coupling}

We have observed in Section 5.1.1 that the function $Y_{\mathbf{k}}(\vartheta)$ we are ultimately interested in has asymptotics for $m R \rightarrow 0$ given by solutions to Eqs. $\left(\mathrm{I}_{ \pm}\right),\left(\mathrm{B}_{ \pm}\right)$. In Section 5.1.2 we introduced a certain class of solutions to these equations. In order to proceed we will now assume that the asymptotics of $Y_{\mathbf{k}}(\vartheta)$ for $m R \rightarrow 0$ can be found within the class of solutions discussed in Section 5.1.2. More precisely, we will assume that for $\mathbf{k}_{ \pm}$chosen as

$$
\mathbf{k}_{ \pm}=\left[ \pm k_{a} \mid a \in \mathbb{K}_{ \pm}\right]
$$

there will exist a distinguished value for $P, P \equiv P(\mathbf{k})$, such that the functions $Y_{\mathbf{k}_{+}, P(\mathbf{k})}^{+}(\vartheta)$ and $Y_{\mathbf{k}_{-}, P(\mathbf{k})}^{-}(\vartheta)$ will both give good approximations to $Y_{\mathbf{k}}(\vartheta)$ for values of $\vartheta$ on the plateau. This assumption can be supported substantially by comparison with the lattice theory where an analogous statement can be checked explicitly [18].

We should keep in mind that $-Q_{\mathbf{k}_{ \pm}, P}^{ \pm}(\vartheta)$ and $Q_{\mathbf{k}_{ \pm}, P}^{ \pm}(\vartheta)$ correspond to the same functions $Y_{\mathbf{k}_{ \pm}, P}^{ \pm}(\vartheta)$, respectively. The requirement that the asymptotics (5.3) provides a good approximation for the values (up to a sign) that the function $Q_{\mathbf{k}}(\vartheta)$ takes on the plateau then implies that $P(\mathbf{k})$ must be a solution to the condition

$$
\Theta\left(\mathbf{k}_{+} \mid P\right)+\Theta\left(\mathbf{k}_{-} \mid P\right) \in \pi \mathbb{Z} .
$$

We will label the different solutions $P$ of (5.6) by an integer $n$. It follows from property (a) that $P_{m}<P_{n}$ if $m<n$. Note furthermore that property (b) above implies that $P_{-n}=-P_{n}$. It follows

\footnotetext{
3 These remarks clarify and correct a previous version of this paper. The author is grateful to W. Nahm for a question which stimulated this discussion.
} 
that the solutions $P_{n}$ and $P_{-n}$ correspond to the same function $Q(\vartheta)$. The solutions $P$ to the quantization conditions are therefore uniquely determined by the data $\left(\mathbf{k}_{+}, \mathbf{k}_{-},|n|\right)$.

Let us furthermore observe that the dependence of $\Theta\left(\mathbf{k}_{ \pm} \mid P\right)$ w.r.t. the variable $R$ is given by

$$
\Theta\left(\mathbf{k}_{ \pm} \mid P\right)=\hat{\Theta}\left(\mathbf{k}_{ \pm} \mid P\right)+4 \delta P|\log m R|,
$$

where $\hat{\Theta}\left(\mathbf{k}_{ \pm} \mid P\right)$ is independent of $R$. This follows from the simple fact that the dependence w.r.t. $m R$ can be removed from Eqs. $\left(\mathrm{I}_{ \pm}\right),\left(\mathrm{B}_{ \pm}\right)$by a shift of the variable $\vartheta$. This circumstance implies that the solutions to the quantization condition (5.6) behave as

$$
P_{n} \sim \frac{n \pi}{8 \delta|\log m R|}\left(1+\mathcal{O}\left((\log m R)^{-1}\right)\right) .
$$

For values of $n$ much smaller than $|\log m R|$ we may observe that the zeros of the function $Q_{\mathbf{k}}(\vartheta)$ which fall into the plateau are separated by a distance of order $|\log m R|$ from each other and from the boundaries of the plateau. The number of these zeros is $|n|-1$. Keeping in mind that the zeros $\vartheta_{a}$ of $Q_{\mathbf{k}}(\vartheta)$ which do not correspond to the rapidities of the left- or right-moving particles are those for which $k_{a}=0$ we are led to identify

$$
|n|-1=n_{\mathrm{o}},
$$

where $n_{\mathrm{o}}$ is the number of indices $a$ such that $k_{a}=0$.

Eqs. (5.5) and (5.8) establish a one-to-one correspondence between the tuples $\mathbf{k}$ which label the solutions $Y_{\mathbf{k}}(\vartheta)$ of (I), (B) and the data $\left(\mathbf{k}_{+}, \mathbf{k}_{-},|n|\right)$ which label the solutions to the quantization conditions (5.6). This means that there is a one-to-one correspondence between the vectors $e_{\mathbf{k}}$ which span $\mathcal{H}_{\mathrm{TBA}}$ and the set of solutions to the quantization conditions (5.6).

\subsubsection{Calculation of the energies}

Let us now observe that the eigenvalues $E_{\mathbf{k}}$ of the Hamiltonian can be calculated explicitly in terms of the data $\left(\mathbf{k}_{+}, \mathbf{k}_{-}, P_{n}\right)$.

\section{Proposition 3. We have}

$$
E_{\mathbf{k}}=\frac{2 \pi}{R}\left(2 P_{n}^{2}-\frac{1}{12}+\sum_{a \in \mathbb{K}_{+}} k_{a}+\sum_{a \in \mathbb{K}_{-}}\left|k_{a}\right|\right) .
$$

The proof of Proposition 3 is given in Appendix C.

To summarize our conclusions: The part of the spectrum contained in $\mathcal{H}_{\text {TBA }}$ has UV asymptotics characterized by formula (5.9) with $P_{n} \equiv P_{n}\left(r \mid \mathbf{k}_{+}, \mathbf{k}_{-}\right)$being defined by (5.6).

\subsubsection{Quantization conditions in the case $\mathbb{K}_{ \pm}=\emptyset$}

Let us consider the quantization conditions (5.6) for the case that $\mathbb{K}_{ \pm}=\emptyset$ where they simplify to

$$
2 \Theta(P) \in \pi \mathbb{Z}
$$

where $\Theta(P) \equiv \Theta\left(\mathbf{k}_{ \pm} \mid P\right)$ for $\mathbb{K}_{ \pm}=\emptyset$.

An explicit formula for the functions $\Theta(P)$ can be extracted from the recent work [11]. This works as follows. A special case of the results of [11] is a simple characterization of the functions $Q^{ \pm}(\vartheta)$ for the case $\mathbb{K}_{ \pm}=\varnothing$ in terms of the solutions to an ordinary differential equation, generalizing similar results for other models which go back to [20,21]. The differential equation 
relevant for the present case is

$$
\left[-\frac{d^{2}}{d x^{2}}-p^{2}+\kappa^{2}\left(e^{2 x}+e^{-2 x / b^{2}}\right)\right] \Psi=0 .
$$

There are unique solutions $\Psi_{ \pm}$to (5.11) which have the asymptotic behavior

$$
\begin{aligned}
& \Psi_{+} \sim \frac{1}{\sqrt{2 \kappa}} \exp \left(\frac{x}{2 b^{2}}-\kappa b^{2} e^{-x / b^{2}}\right) \quad \text { for } x \rightarrow-\infty, \\
& \Psi_{-} \sim \frac{1}{\sqrt{2 \kappa}} \exp \left(-\frac{x}{2}-\kappa e^{x}\right) \quad \text { for } x \rightarrow+\infty,
\end{aligned}
$$

respectively. The functions $Q^{ \pm}(\vartheta)$ are then simply given as

$$
Q^{+}(\vartheta) \equiv Q^{-}(-\vartheta) \equiv \Psi_{+} \frac{d}{d x} \Psi_{-}-\Psi_{-} \frac{d}{d x} \Psi_{+},
$$

provided that we identify the respective parameters as follows, ${ }^{4}$

$$
\kappa=-\frac{\kappa_{0}}{2 \sin \frac{\pi b^{2}}{1+b^{2}}} \frac{m R}{2} e^{\vartheta}, \quad \kappa_{0}=-\frac{2 \sqrt{\pi}}{\Gamma\left(-\frac{1}{2\left(1+b^{2}\right)}\right) \Gamma\left(1-\frac{b^{2}}{2\left(1+b^{2}\right)}\right)}, \quad b p=2 P .
$$

The characterization of $Q^{ \pm}(\vartheta)$ in terms of the ODE (5.11) allowed the authors of [11] to determine the asymptotics of $Q^{ \pm}(\vartheta)$. The explicit expression for $e^{2 i \Theta(P)}$ which follows from formula (177) in [11] is given by the formula

$$
e^{2 i \Theta(P)}=-\rho^{-8 i \delta P} \frac{\Gamma(1+2 i b P) \Gamma\left(1+2 i b^{-1} P\right)}{\Gamma(1-2 i b P) \Gamma\left(1-2 i b^{-1} P\right)},
$$

in which we have used the abbreviation

$$
\rho \equiv \frac{R}{2 \pi} \frac{m}{4 \sqrt{\pi}} \Gamma\left(\frac{1}{2+2 b^{2}}\right) \Gamma\left(1+\frac{b^{2}}{2+2 b^{2}}\right) .
$$

Having the explicit formula (5.15) at hand, we may easily verify that the conditions (a), (b) as formulated in Claim 4 are satisfied for this case.

\subsection{Quantization conditions from Liouville theory}

The basic idea [17] is that the quantum Hamiltonian of the sinh-Gordon model can be represented in the following schematic form

$$
\mathbf{H}_{\mathrm{ShG}}=\frac{2 \pi}{R} \int_{0}^{2 \pi} d \sigma\left\{4 \pi \Pi^{2}+\frac{1}{16 \pi}\left(\partial_{\sigma} \varphi\right)^{2}+\left(\frac{R}{2 \pi}\right)^{4 b \delta} \mu\left(e^{b \varphi}+e^{-b \varphi}\right)\right\} .
$$

For $R \rightarrow 0$ one observes that in configuration space there is a large region

$$
-4 \delta \log \frac{2 \pi}{R}+\frac{1}{b} \log \mu \ll \varphi
$$

where one can neglect the interaction term $e^{-b \varphi}$. Dropping this term yields the Hamiltonian $\mathbf{H}_{\mathrm{L}}$ of quantum Liouville theory.

\footnotetext{
4 Concerning the comparison with [11] let us note that the parameter $n$ used there is related to $b^{2}$ via $n=2 / b^{2}$.
} 


\subsubsection{The spectrum of Liouville theory}

Let us quickly review the relevant aspects of the quantum Liouville theory as constructed in [22], see [23] for a review and further references. The spectrum of $\mathbf{H}_{\mathrm{L}}$ may be represented in the form

$$
\mathcal{H}_{\mathrm{L}}=\int_{0}^{\infty} d P \mathcal{F}_{P}^{+} \otimes \mathcal{F}_{P}^{-}
$$

where $\mathcal{F}_{P}^{ \pm} \simeq \mathcal{F}^{ \pm}$for all $P \in \mathbb{R}_{+}$, with $\mathcal{F}^{ \pm}$being standard Fock spaces generated by oscillators $a_{k}^{ \pm},\left[a_{k}^{ \pm}, a_{l}^{ \pm}\right]=\frac{k}{2} \delta_{k+l, 0}$ from the Fock vacua $\Omega^{ \pm}$. The spaces $\mathcal{F}_{P}^{ \pm}$have bases spanned by the vectors

$$
e_{P, \mathbf{k}}^{ \pm}=a_{-k_{M}}^{ \pm} \cdots a_{-k_{1}}^{ \pm} \Omega^{ \pm}, \quad \mathbf{k}=\left[k_{1}, \ldots, k_{M}\right] .
$$

The spaces $\mathcal{F}_{P}^{ \pm}$carry an irreducible representation of two commuting copies of the Virasoro algebra with generators

$$
\begin{aligned}
& L_{n}^{ \pm}(P)=2(P+i n \delta) a_{n}+\sum_{k \neq 0, n} a_{k}^{ \pm} a_{n-k}^{ \pm}, \\
& L_{0}^{ \pm}(P)=P^{2}+\delta^{2}+2 \sum_{k>0} a_{-k}^{ \pm} a_{k}^{ \pm} .
\end{aligned}
$$

The central charge $c$ is given in terms of $\delta$ by the formula

$$
c=1+24 \delta^{2} \text {. }
$$

The action of the Hamiltonian $\mathbf{H}_{\mathrm{L}}$ of quantum Liouville theory on $\mathcal{F}_{P}^{+} \otimes \mathcal{F}_{P}^{-}$is then represented by

$$
\mathbf{H}_{\mathrm{L}}(P)=\frac{2 \pi}{R}\left(L_{0}^{+}(P)+L_{0}^{-}(P)-\frac{c}{12}\right)
$$

which means that the states $e_{P, \mathbf{k}_{+}}^{+} \otimes e_{P, \mathbf{k}_{-}}^{-}$have eigenvalue given by

$$
\mathbf{H}_{\mathrm{L}}(P) \cdot e_{P, \mathbf{k}_{+}}^{+} \otimes e_{P, \mathbf{k}_{-}}^{-}=\frac{2 \pi}{R}\left(2 P^{2}-\frac{1}{12}+\sum_{a=1}^{M_{+}} k_{a}^{+}+\sum_{a=1}^{M_{-}} k_{a}^{-}\right) e_{P, \mathbf{k}_{+}}^{+} \otimes e_{P, \mathbf{k}_{-}}^{-} .
$$

\subsubsection{The reflection operator}

For $R \rightarrow 0$ there is a large region in the configuration space of Liouville theory

$$
\varphi \ll 4 \delta \log \frac{2 \pi}{R}-\frac{1}{b} \log \mu
$$

within which one expects that it should be possible to describe the states of the Liouville theory in terms of a Schrödinger representation for the zero mode $\varphi_{0}=\int_{0}^{2 \pi} d \sigma \varphi$, see [23] for a discussion of scope and limitations of such a representation. Eigenstates $\Psi_{P}$ would then be represented by means of wave-functions $\Psi\left(\varphi_{0}\right) \in \mathcal{F}^{+} \otimes \mathcal{F}^{-}$which have the form

$$
\Psi\left(\varphi_{0}\right)=\left(\frac{R}{2 \pi}\right)^{4 i \delta P} e^{i P \varphi_{0}} \mathfrak{F}_{P}+\left(\frac{R}{2 \pi}\right)^{-4 i \delta P} e^{-i P \varphi_{0}}\left(\mathbf{T}(P) \cdot \mathfrak{F}_{P}\right),
$$


where $\mathfrak{F}_{P} \in \mathcal{F}^{+} \otimes \mathcal{F}^{-}$, and $\mathbf{T}(P)$ is the so-called reflection operator which describes the reflection by the Liouville interaction $e^{b \varphi}$ which remains in (5.17) after one has neglected $e^{-b \varphi}$ $[17,23]$.

The operator $\mathbf{T}(P): \mathcal{F}^{+} \otimes \mathcal{F}^{-} \rightarrow \mathcal{F}^{+} \otimes \mathcal{F}^{-}$is fully characterized by the following properties:

- The operator $\mathbf{T}(P)$ can be factorized as

$$
\mathbf{T}(P)=T(P) \mathbf{T}^{+}(P) \otimes \mathbf{T}^{-}(P) .
$$

- The operators $\mathbf{T}^{ \pm}(P)$ are completely defined by the intertwining property

$$
\mathbf{T}^{ \pm}(P) \cdot L_{n}^{ \pm}(P)=L_{n}^{ \pm}(-P) \cdot \mathbf{T}^{ \pm}(P),
$$

together with the requirements that

$$
\begin{aligned}
& \left(\mathbf{T}^{+}(P) \otimes 1\right) \cdot\left(\Omega^{+} \otimes \Omega^{-}\right)=\left(\Omega^{+} \otimes \Omega^{-}\right), \\
& \left(1 \otimes \mathbf{T}^{-}(P)\right) \cdot\left(\Omega^{+} \otimes \Omega^{-}\right)=\left(\Omega^{+} \otimes \Omega^{-}\right) .
\end{aligned}
$$

- The reflection amplitude $T(P)$ is given by the formula $[17,24]$

$$
T(P)=-\left(\pi \mu \gamma\left(b^{2}\right)\right)^{-2 i P / b} \frac{\Gamma(1+2 i b P) \Gamma\left(1+2 i b^{-1} P\right)}{\Gamma(1-2 i b P) \Gamma\left(1-2 i b^{-1} P\right)} .
$$

Note in particular that the unitary operators $\mathbf{T}^{ \pm}(P)$ preserve the subspaces of $\mathcal{F}^{ \pm}$defined by $\sum_{a=1}^{M_{ \pm}} k_{a}^{ \pm}=$const. We may therefore introduce eigenstates $\mathfrak{f}^{ \pm}\left(\mathbf{k}_{ \pm} \mid P\right) \in \mathcal{F}^{ \pm}$of $\mathbf{T}^{ \pm}(P)$ which will form bases for $\mathcal{F}^{ \pm}$when $\mathbf{k}_{ \pm}$vary over all tuples $\left[k_{1}^{ \pm}, \ldots, k_{M_{ \pm}}^{ \pm}\right]$of integers with $k_{a}^{ \pm}>0$. The corresponding eigenvalues will be written as $\exp \left(i \theta_{ \pm}\left(\mathbf{k}_{ \pm} \mid P\right)\right)$.

\subsubsection{Quantization conditions of the sinh-Gordon model in the UV}

In the case of the sinh-Gordon model one would expect that a representation for the eigenstates of the Hamiltonian in the form (5.26) should be possible in the region

$$
-4 \delta \log \frac{2 \pi}{R}+\frac{1}{b} \log \mu \ll \varphi \ll 4 \delta \log \frac{2 \pi}{R}-\frac{1}{b} \log \mu .
$$

However, as opposed to the Liouville case one may no longer choose $P$ arbitrarily. One way to find the restrictions on $P$ is to observe that the wave-functions of energy eigenstates in the parity symmetric potential $\cosh (b \varphi)$ should have definite parity \pm 1 . The ground-state, in particular, should have positive parity. Imposing this requirement on (5.26) with $\mathfrak{F}_{P}$ being chosen as $\mathfrak{f}^{+}\left(\mathbf{k}_{+} \mid P\right) \otimes \mathfrak{f}^{-}\left(\mathbf{k}_{-} \mid P\right)$ leads to the quantization conditions

$$
\arg T(P)+\theta_{+}\left(\mathbf{k}_{+} \mid P\right)+\theta_{-}\left(\mathbf{k}_{-} \mid P\right)-8 \delta P \log (R / 2 \pi) \in \pi \mathbf{Z},
$$

where $\theta_{ \pm}\left(\mathbf{k}_{ \pm} \mid P\right)$ are the phases of the eigenvalues of $\mathbf{T}^{ \pm}(P)$ as introduced above. Introducing a positive integer $m$ which labels the solutions $P_{m}$ of (5.31) we are lead to the conclusion that the UV asymptotics for the spectrum of the sinh-Gordon model is given by

$$
E\left(\mathbf{k}_{+}, \mathbf{k}_{-}, m\right)=\frac{2 \pi}{R}\left(2 P_{m}^{2}-\frac{1}{12}+\sum_{a \in \mathbb{K}_{+}} k_{a}+\sum_{a \in \mathbb{K}_{-}} k_{a}\right),
$$

with $P_{m}=P_{m}\left(\mathbf{k}_{+}, \mathbf{k}_{-}\right)$being a solution to (5.31). We observe a remarkable agreement with the description of $\mathcal{H}_{\mathrm{TBA}}$ that we had found in Section 5.1. 
Comparing formulae (5.15) and (5.29) we furthermore observe that the quantization conditions (5.31) and (5.6) precisely agree in the case $\mathbb{K}_{ \pm}=\emptyset$ provided that the parameters $m$ and $\mu$ are related as

$$
\frac{\pi \mu}{\gamma\left(-b^{2}\right)}=-\left[\frac{m}{4 \sqrt{\pi}} \Gamma\left(\frac{1}{2+2 b^{2}}\right) \Gamma\left(1+\frac{b^{2}}{2+2 b^{2}}\right)\right]^{2+2 b^{2}} .
$$

These observations not only represent a highly nontrivial confirmation of the picture proposed in this paper, they also lead to a re-derivation of the important result from [6] concerning the exact relation between the scale parameters $\mu$ and $m$ defined in terms of the UV and the IR behavior of the sinh-Gordon model, respectively. that

On the other hand we would like to point out that our discussion above leads us to conjecture

$$
2 \Theta\left(\mathbf{k}_{ \pm} \mid P\right)=T(P)+2 \theta_{ \pm}\left(\mathbf{k}_{ \pm} \mid P\right) .
$$

This relation would represent a remarkable and highly nontrivial link between the integrable structure of quantum $\mathrm{KdV}$ theory with $c>1$-as encoded in $\Theta\left(\mathbf{k}_{ \pm} \mid P\right)$-and the conformal structure of quantum Liouville theory that was used in the definition of $\theta_{ \pm}\left(\mathbf{k}_{ \pm} \mid P\right)$, respectively.

\section{Concluding remarks}

The completeness of $\mathcal{H}_{\mathrm{TBA}}$ within $\mathcal{H}_{\mathrm{SG}}$ (Conjecture 1) may further be checked by an analysis of the semiclassical limit $b \rightarrow 0$. This is currently under investigation. Our preliminary results seem to support Conjecture 1.

It seems natural to compare our results to the description of the Sine-Gordon spectrum in terms of nonlinear integral equations (NLIE) that was found by Destri and De Vega in [25-27], see also [28]. A quick comparison reveals important differences between the two cases, however. We note, in particular, that the functions constrained by the respective nonlinear integral equations are related to the Q-functions in different ways, as can be inferred from [19,29]. In the case of the sinh-Gordon model one would have to look for a nonlinear integral equation satisfied by the function

$$
a(u)=\frac{q(u+i b)}{q(u-i b)},
$$

in order to be able to compare the NLIE of Destri-De Vega type more directly to the NLIE which we have discussed here. It should be very illuminating to analyze in some detail how the physical differences between the two models are encoded in the analytic structure of the $Q$-functions that characterize the respective spectra.

\section{Appendix A. The function $D_{\alpha}(x)$}

In the main text we have been using the function $D_{\alpha}(x)$ which may be defined by the following integral representation

$$
D_{\alpha}(x)=\exp \left\{i \int_{\mathbb{R}+i 0} \frac{d t}{2 t} \frac{\cos (2 t x) \sin (2 \alpha t)}{\sinh b t \sinh \frac{t}{b}}\right\} .
$$

$D_{\alpha}(x)$ is a meromorphic function with zeros at $x \in \Upsilon_{-\alpha}$ and poles at $x \in \Upsilon_{\alpha}$. The function $D_{\alpha}(x)$ is self-dual in $b$ (but we will omit this index) and has the following properties 


$$
\begin{array}{lll}
\text { functional equation } & \frac{D_{\alpha}\left(x+\frac{i}{2} b^{ \pm 1}\right)}{D_{\alpha}\left(x-\frac{i}{2} b^{ \pm 1}\right)}=\frac{\cosh \pi b^{ \pm 1}(x+\alpha)}{\cosh \pi b^{ \pm 1}(x-\alpha)}, \\
x \text {-parity } & D_{\alpha}(x)=D_{\alpha}(-x), \\
\text { reflection property } & D_{\alpha}(x) D_{-\alpha}(x)=1, \\
\text { complex conjugation } & \overline{D_{\alpha}(x)}=D_{-\bar{\alpha}}(\bar{x}), \\
x \text {-asymptotics } & D_{\alpha}(x) \sim \begin{cases}e^{-2 \pi i \alpha x} & \text { for }|x| \rightarrow \infty,|\arg (x)|<\frac{\pi}{2}, \\
e^{+2 \pi i \alpha x} & \text { for }|x| \rightarrow \infty,|\arg (x)|>\frac{\pi}{2},\end{cases} \\
\alpha \text {-asymptotics } & D_{\alpha}(x) \sim \begin{cases}e^{-i \pi\left(x^{2}+\alpha^{2}+\frac{1}{12}\left(b^{2}+b^{-2}\right)\right)} & \text { if }|\alpha| \rightarrow \infty,|\arg (\alpha)|<\frac{\pi}{2}, \\
e^{+i \pi\left(x^{2}+\alpha^{2}+\frac{1}{12}\left(b^{2}+b^{-2}\right)\right)} & \text { if }|\alpha| \rightarrow \infty,|\arg (\alpha)|>\frac{\pi}{2} .\end{cases}
\end{array}
$$

$D_{\alpha}(x)$ is related to the so-called quantum dilogarithm function $w_{b}(x)$ as follows:

$$
D_{\alpha}(x)=\frac{w_{b}(x+\alpha)}{w_{b}(x-\alpha)} .
$$

We refer to [10] for more properties of these remarkable special functions and references to the original literature.

\section{Appendix B. Proof of Theorem 1}

The main tool for the proof of Theorem 1 will be the following proposition.

Proposition 4. Assume that the function $Y(\vartheta)$ satisfies $(\mathrm{I})_{\mathrm{L}}$ and has the properties $\left(\mathrm{Y}_{1}\right)_{\mathrm{L}},\left(\mathrm{Y}_{2}\right)_{\mathrm{L}}$ and $\left(\mathrm{Y}_{3}\right)_{\mathrm{L}}$. The function $Q(\vartheta)$ defined in Eq. $(\mathrm{Q})_{\mathrm{L}}$ then satisfies Eq. (2.5) and has the properties $\left(\mathrm{Q}_{1}\right)_{\mathrm{L}}-\left(\mathrm{Q}_{3}\right)_{\mathrm{L}}$ above.

Proof. As a preparation, and in order to prove property $\left(\mathrm{Q}_{1}\right)_{\mathrm{L}}$ let us note that

$$
\log (W(\vartheta)+Y(\vartheta)) \sim-i \frac{2}{\pi} \delta^{2} \mathrm{~N}\left(\vartheta \mp \sigma \mp i \frac{\pi}{2}\right)^{2} \text { for }|u| \rightarrow \infty,|\arg ( \pm u)|<\frac{\pi}{2} .
$$

It follows that the limit $\vartheta \rightarrow \pm \infty$ cannot simply be exchanged with the integration in $(\mathrm{Q})_{\mathrm{L}}$. The asymptotics $\vartheta \rightarrow \pm \infty$ is instead dominated by the part of the integration region where $\left|\vartheta^{\prime}-\vartheta\right|=$ $\mathcal{O}(1)$. Changing variables to $\vartheta^{\prime \prime}=\vartheta^{\prime}-\vartheta$ and using (B.1) then allows us to verify $\left(\mathrm{Q}_{1}\right)_{\mathrm{L}}$.

In order to prove property $\left(\mathrm{Q}_{2}\right)_{\mathrm{L}}$ and $\left(\mathrm{Q}_{3}\right)_{\mathrm{L}}$ it is useful to consider

$$
\begin{aligned}
\left(\mathrm{Q}^{\prime}\right)_{\mathrm{L}} \quad \frac{d}{d \vartheta} \log Q(\vartheta)= & \int_{\mathcal{C}} \frac{d \vartheta^{\prime}}{4 \pi} \frac{1}{\cosh \left(\vartheta-\vartheta^{\prime}\right)} \frac{d}{d \vartheta^{\prime}} \log \left(W\left(\vartheta^{\prime}\right)+Y\left(\vartheta^{\prime}\right)\right) \\
& -\frac{N}{2}\left(\frac{1}{\cosh (\vartheta-\sigma)}-\frac{1}{\cosh (\vartheta+\sigma)}\right) \\
& +\sum_{a=1}^{M^{\prime}} \frac{1}{\sinh \left(\vartheta-\vartheta_{a}\right)}+\frac{1}{2} \sum_{a=M^{\prime}+1}^{M} \frac{1}{\sinh \left(\vartheta-\vartheta_{a}\right)} .
\end{aligned}
$$

It is clear that $\frac{d}{d \vartheta} \log Q(\vartheta)$ is meromorphic, being the convolution of two meromorphic functions. Poles and zeros of $Q$ correspond to poles of $\frac{d}{d \vartheta} \log Q(\vartheta)$ with residue giving the order of the 
corresponding pole or zero of $Q$. Besides the poles which are explicit in $\left(\mathrm{Q}^{\prime}\right)_{\mathrm{L}}$ we will find additional poles whenever the contour $\mathcal{C}$ of integration gets pinched between the poles from $1 / \cosh \left(\vartheta-\vartheta^{\prime}\right)$ and those of $\frac{d}{d \vartheta^{\prime}} \log \left(W\left(\vartheta^{\prime}\right)+Y\left(\vartheta^{\prime}\right)\right)$. The latter arise on the one hand from the poles of $W\left(\vartheta^{\prime}\right)+Y\left(\vartheta^{\prime}\right)$ as specified in $\left(\mathrm{Y}_{2}\right)_{\mathrm{L}}$, and on the other hand from the zeros of $W\left(\vartheta^{\prime}\right)+$ $Y\left(\vartheta^{\prime}\right)$ as specified in $\left(\mathrm{Y}_{3}\right)_{\mathrm{L}}$. Note in particular that the pole of $W\left(\vartheta^{\prime}\right)+Y\left(\vartheta^{\prime}\right)$ at $\sigma \pm \vartheta=0$ comes only from the function $W\left(\vartheta^{\prime}\right)$ and therefore has order $\mathrm{N}$. The verification of $\left(\mathrm{Q}_{2}\right)_{\mathrm{L}}$ and $\left(\mathrm{Q}_{3}\right)_{\mathrm{L}}$ is now straightforward.

In order to prove Eq. (2.5) note that $\log Q\left(\vartheta+i \frac{\pi}{2}\right)+\log Q\left(\vartheta-i \frac{\pi}{2}\right)$ can be represented as

$$
\lim _{\epsilon \rightarrow 0} \int_{\mathcal{C}} \frac{d \vartheta^{\prime}}{4 \pi i}\left[\frac{1}{\sinh \left(\vartheta-\vartheta^{\prime}-i \epsilon\right)}-\frac{1}{\sinh \left(\vartheta-\vartheta^{\prime}+i \epsilon\right)}\right] \log \left(W\left(\vartheta^{\prime}\right)+Y\left(\vartheta^{\prime}\right)\right),
$$

as all other terms drop out. Validity of Eq. (2.5) now follows easily.

Proposition 4 allows us to complete the proof of Theorem 1 as follows.

Let $Y \in \mathcal{Y}_{M}$. Define $Q(\vartheta)$ by Eq. (Q) $)_{\mathrm{L}}$. It then follows from Proposition 4 that $Q(\vartheta)$ satisfies the properties $\left(\mathrm{Q}_{1}\right)_{\mathrm{L}}-\left(\mathrm{Q}_{3}\right)_{\mathrm{L}}$. In order to prove $(\mathrm{Wr})_{\mathrm{L}}$ it suffices to observe that Eq. $(\mathrm{Q})_{\mathrm{L}}$ allows us to write the integral equation $(\mathrm{I})_{\mathrm{L}}$ as

$$
\log Y(\vartheta)=\log Q(\vartheta+i \tau)+\log Q(\vartheta-i \tau) .
$$

The functional relation $(\mathrm{Wr})_{\mathrm{L}}$ follows by combining (2.5) and (B.3).

Conversely, let $\tilde{Q} \in \mathcal{Q}$. Define $Y(\vartheta)$ by

$$
W(\vartheta)+Y(\vartheta)=\tilde{Q}\left(\vartheta+i \frac{\pi}{2}\right) \tilde{Q}\left(\vartheta-i \frac{\pi}{2}\right) .
$$

It is easy to see that the properties of $\tilde{Q}$ imply that $Y(\vartheta)$ satisfies the properties $\left(\mathrm{Y}_{1}\right)_{\mathrm{L}},\left(\mathrm{Y}_{2}\right)_{\mathrm{L}}$ and $\left(\mathrm{Y}_{3}\right)_{\mathrm{L}}$ with set $\mathbf{Z}=\left\{\vartheta_{1}, \ldots, \vartheta_{M}\right\}$ being the set of zeros of $\tilde{Q}(\vartheta)$ in $\mathbb{S}$. Let us introduce the function $R(\vartheta)=\log \tilde{Q}(\vartheta)-\log Q(\vartheta)$, where $Q(\vartheta)$ is defined by Eq. (Q) $)_{\mathrm{L}}$. We want to show that $R(\vartheta) \equiv 0$. This will be done in two steps.

Lemma 3. The function $R(\vartheta)$ has the following properties:

(i) $R(\vartheta)$ is entire analytic,

(ii) $R(\vartheta) \sim$ const for $|\vartheta| \rightarrow \infty,|\arg ( \pm \vartheta)|<\frac{\pi}{2}$,

(iii) $R(\vartheta)$ solves the difference equation $R\left(\vartheta+i \frac{\pi}{2}\right)+R\left(\vartheta-i \frac{\pi}{2}\right)=0$.

Proof. It follows from Proposition 4 that both $Q$ and $\tilde{Q}$ have poles of order $\mathrm{N}$ at $s \pm u=\frac{i}{2} \delta$. The functions $\tilde{Q}$ and $Q$ also have the same set of zeros $\mathbf{Z}=\left\{\vartheta_{1}, \ldots, \vartheta_{M}\right\}$ within $\mathbb{S}$. It follows that $R(\vartheta)$ is analytic for all $\vartheta \in \mathbb{S}$. Property (ii) follows directly by comparing $\left(\mathrm{Q}_{1}\right)_{\mathrm{L}}$ and $\left(\mathrm{Q}_{1}^{\prime}\right)_{\mathrm{L}}$. Property (iii) is clear from (2.5) and (B.4). This property finally allows us to prove that $R(\vartheta)$ is entire analytic from the fact that it is analytic in $\mathbb{S}$.

Lemma 4. The only function $R(\vartheta)$ with the properties (i)-(iii) formulated in Lemma 3 is $R(\vartheta) \equiv 0$.

Proof. Property (iii) implies that $R(\vartheta)$ is periodic with period $2 \pi i$. It therefore follows from (i), (iii) that $R(\vartheta)=r\left(e^{\vartheta}\right)$, with $r(z)$ being analytic on $\mathbb{P}^{1} \backslash\{0, \infty\}$. Taking into account that $r(z)$ is 
bounded at $0, \infty$ by property (ii) allows us to conclude that $r(z)$ must be a constant, which can only be zero by property (iii).

Inserting the representation for $\tilde{Q}(\vartheta)$ that is given by Eq. $(\mathrm{Q})_{\mathrm{L}}$ into relation $(\mathrm{Wr})_{\mathrm{L}}$ then shows that $Y(\vartheta)$ must satisfy the integral equation $(\mathrm{I})_{\mathrm{L}}$. This finishes the proof of Theorem 1 .

\section{Appendix C. Proof of Proposition 3}

To begin with, let us note that summing over $a$ in the relations $\left(\mathrm{B}_{ \pm}\right)$yields the relations

$$
\pm \frac{m R}{2} \sum_{a \in \mathbb{K}_{ \pm}} e^{ \pm \vartheta_{a}}-\int_{\mathbb{R}} \frac{d \vartheta}{2 \pi} \log \left(1+Y_{\mathbf{k}_{ \pm}^{ \pm}}^{ \pm}(\vartheta)\right) \sum_{a \in \mathbb{K}_{ \pm}} \tau\left(\vartheta_{a}-\vartheta\right)=2 \pi \sum_{a \in \mathbb{K}_{ \pm}} k_{a} .
$$

Inserting the expressions for $\sum_{a \in \mathbb{K}_{ \pm}} \tau\left(\vartheta_{a}-\vartheta\right)$ which follow from ( $\left.\mathrm{I}_{ \pm}\right)$leads to the formula

$$
\pm \frac{m R}{2}\left(\sum_{a \in \mathbb{K}_{ \pm}} e^{ \pm \vartheta_{a}}-\int_{\mathbb{R}} \frac{d \vartheta}{2 \pi} e^{ \pm \vartheta} \log \left(1+Y_{\mathbf{k}_{ \pm}^{ \pm}}^{ \pm}(\vartheta)\right)\right)=2 \pi \sum_{a \in \mathbb{K}_{ \pm}} k_{a}+\mathcal{I}_{\mathbf{k}_{ \pm}}^{ \pm}
$$

where $\mathcal{I}_{\mathbf{k}_{ \pm}}^{ \pm}$is defined as

$$
\begin{aligned}
\mathcal{I}_{\mathbf{k}_{ \pm}}^{ \pm} \equiv & \int_{\mathbb{R}} \frac{d \vartheta}{2 \pi} \log \left(1+Y_{\mathbf{k}_{ \pm}}^{ \pm}(\vartheta)\right) \frac{\partial}{\partial \vartheta}\left(\log Y_{\mathbf{k}_{ \pm}^{ \pm}}(\vartheta)\right. \\
& \left.-\int_{\mathbb{R}} \frac{d \vartheta^{\prime}}{2 \pi} \sigma\left(\vartheta-\vartheta^{\prime}\right) \log \left(1+Y_{\mathbf{k}_{ \pm}^{ \pm}}^{ \pm}\left(\vartheta^{\prime}\right)\right)\right) .
\end{aligned}
$$

This integral can be evaluated explicitly:

\section{Proposition 5.}

$$
\mathcal{I}_{\mathbf{k}_{ \pm}^{ \pm}}^{ \pm}= \pm 2 \pi\left(P^{2}-\frac{1}{24}\right)
$$

Proof. We note that the integrals defining $\mathcal{I}_{\mathbf{k}_{ \pm}}^{ \pm}$are absolutely convergent. We may therefore perform the calculation under the assumption that $\Im(P)>0$ and take the limit $\Im(P) \rightarrow 0$ in the end.

Let us first consider

$$
\int_{-\xi}^{\xi} \frac{d \vartheta}{2 \pi} \int_{-\zeta}^{\zeta} \frac{d \vartheta^{\prime}}{2 \pi} F(\vartheta) \frac{\partial}{\partial \vartheta} \sigma\left(\vartheta-\vartheta^{\prime}\right) F\left(\vartheta^{\prime}\right)
$$

where $F(\vartheta) \equiv \log \left(1+Y_{\mathbf{k}_{+}}^{+}(\vartheta)\right)$. The anti-symmetry of the kernel $\frac{\partial}{\partial \vartheta} \sigma\left(\vartheta-\vartheta^{\prime}\right)$ in (C.4) implies that the integral (C.4) can be written as

$$
\int_{-\xi}^{\xi} \frac{d \vartheta}{2 \pi} F(\vartheta)\left(\int_{-\zeta}^{-\xi} \frac{d \vartheta^{\prime}}{2 \pi} \frac{\partial}{\partial \vartheta} \sigma\left(\vartheta-\vartheta^{\prime}\right) F\left(\vartheta^{\prime}\right)+\int_{\xi}^{\zeta} \frac{d \vartheta^{\prime}}{2 \pi} \frac{\partial}{\partial \vartheta} \sigma\left(\vartheta-\vartheta^{\prime}\right) F\left(\vartheta^{\prime}\right)\right) .
$$


We will ultimately send $\xi, \zeta \rightarrow \infty$ such that $0<\xi<\zeta$. This allows us to approximate $F\left(\vartheta^{\prime}\right)$ in (C.5) by its asymptotics

$$
F\left(\vartheta^{\prime}\right) \sim \begin{cases}0 & \text { for } \vartheta^{\prime} \rightarrow \infty, \\ 8 i \delta P \vartheta^{\prime} & \text { for } \vartheta^{\prime} \rightarrow-\infty,\end{cases}
$$

which leads us to represent the integral in (C.5) as

$$
\frac{4 i \delta}{\pi} P \int_{-\xi}^{\xi} \frac{d \vartheta}{2 \pi} F(\vartheta)(\xi \sigma(\vartheta+\xi)-\zeta \sigma(\vartheta+\zeta)-\arg S(\vartheta+\xi)+\arg S(\vartheta+\zeta)) .
$$

Noting that $\sigma(\vartheta)$ and $\arg S(\vartheta)$ are peaked around $\vartheta=0$ allows us to use the asymptotics (C.6) for $F(\vartheta)$, leading to us to approximate (C.5) by

$$
\frac{(4 \delta P)^{2}}{\pi^{2}} \int_{0}^{2 \xi} d \vartheta\left(\xi^{2} \sigma(\vartheta)+\vartheta \arg S(\vartheta)\right)
$$

\section{Lemma 5.}

$$
\int_{\mathbb{R}} d \vartheta \sigma(\vartheta)=2 \pi, \quad \int_{\mathbb{R}} d \vartheta \vartheta^{2} \sigma(\vartheta)=2 \pi \frac{\pi^{2}}{(2 \delta)^{2}}
$$

Proof. The lemma is proved by replacing the contour of integration $\mathbb{R}$ by $\frac{1}{2}(\mathbb{R}+(\mathbb{R}+i \pi))+$ $\frac{1}{2}(\mathbb{R}-(\mathbb{R}-i \pi))$. Integrating over $\mathbb{R}+i \pi$ one may use that $\sigma(\vartheta+\pi)=-\sigma(\vartheta)$. The contour $\frac{1}{2}(\mathbb{R}-(\mathbb{R}-i \pi))$ may be closed at infinity, and the corresponding contribution evaluated by the residue theorem.

It follows from (C.8) together with Lemma 5 that the leading asymptotics of (C.5) for $\xi \rightarrow \infty$ is given by the terms

$$
\frac{(4 \delta P)^{2}}{\pi} \xi^{2}-2 \pi P^{2}
$$

It remains to consider the first term in the definition of $\mathcal{I}_{\mathbf{k}_{+}}^{+}$, regularized as

$$
\begin{aligned}
\int_{-\xi}^{\xi} & \frac{d \vartheta}{2 \pi} \log \left(1+Y_{\mathbf{k}_{+}}^{+}(\vartheta)\right) \frac{\partial}{\partial \vartheta} \log Y_{\mathbf{k}_{+}}^{+}(\vartheta) \\
& =\int_{Y_{\mathbf{k}_{+}}^{(-\xi)}}^{Y_{\mathbf{k}_{+}}^{+}(\xi)} \frac{d Y}{2 \pi} \frac{\log (1+Y)}{Y} \\
& =-\int_{0}^{\log Y_{\mathbf{k}_{+}}^{+}(-\xi)} \frac{d x}{2 \pi}\left(x+\log \left(1+e^{-x}\right)\right)-\int_{\log Y_{\mathbf{k}_{+}^{+}}^{+}(\xi)}^{0} \frac{d x}{2 \pi} \log \left(1+e^{x}\right) .
\end{aligned}
$$


In the limit when $\xi \rightarrow \infty$ we may note that (C.6) implies that $\log Y_{\mathbf{k}_{+}}^{+}(\xi) \sim \log F(\vartheta) \rightarrow-\infty$, whereas $\log Y_{\mathbf{k}_{+}}^{+}(-\xi) \sim-8 i \delta P \xi$. We therefore may estimate the leading behavior of (C.10) as

$$
\int_{-\xi}^{\xi} \frac{d \vartheta}{2 \pi} \log \left(1+Y_{\mathbf{k}_{+}}^{+}(\vartheta)\right) \frac{\partial}{\partial \vartheta} \log Y_{\mathbf{k}_{+}^{+}}(\vartheta) \sim \frac{(4 \delta P)^{2}}{\pi} \xi^{2}-2 \int_{0}^{1} \frac{d Y}{2 \pi} \frac{\log (1+Y)}{Y} .
$$

Subtracting (C.9) from (C.11) and using $\int_{0}^{1} \frac{d Y}{2 \pi} \frac{\log (1+Y)}{Y}=\frac{\pi}{24}$ concludes the proof of the proposition for the case of $\mathcal{I}_{\mathbf{k}_{+}}^{+}$. The case of $\mathcal{I}_{\mathbf{k}_{-}}^{-}$can be treated in a similar way.

Inserting formula (C.3) into (C.1) concludes the proof of Proposition 3.

\section{References}

[1] S.N. Vergeles, V.M. Gryanik, Two-dimensional quantum field theories which admit exact solutions, Sov. J. Nucl. Phys. 23 (1976) 704-709.

[2] A. Fring, G. Mussardo, P. Simonetti, Form-factors for integrable Lagrangian field theories, the sinh-Gordon theory, Nucl. Phys. B 393 (1993) 413-441.

[3] A. Koubek, G. Mussardo, On the operator content of the sinh-Gordon model, Phys. Lett. B 311 (1993) $193-201$.

[4] V. Brazhnikov, S. Lukyanov, Angular quantization and form factors in massive integrable models, Nucl. Phys. B 512 (1998) 616-636.

[5] G. Lechner, An existence proof for interacting quantum field theories with a factorising S-matrix, Commun. Math. Phys. 277 (3) (2008) 821-860.

[6] Al.B. Zamolodchikov, Mass scale in the sine-Gordon model and its reductions, Int. J. Mod. Phys. A 10 (1995) 1125-1150.

[7] Al.B. Zamolodchikov, Resonance factorized scattering and roaming trajectories, J. Phys. A 39 (41) (2006) 1284712861.

[8] Al.B. Zamolodchikov, On the thermodynamic Bethe ansatz equation in sinh-Gordon model, J. Phys. A 39 (41) (2006) 12847-12861.

[9] S. Lukyanov, Finite temperature expectation values of local fields in the sinh-Gordon model, Nucl. Phys. B 612 (2001) 391-412.

[10] A. Bytsko, J. Teschner, Quantization of models with non-compact quantum group symmetry. Modular XXZ magnet and lattice sinh-Gordon model, J. Phys. A 39 (2006) 12927-12981.

[11] V.A. Fateev, S.L. Lukyanov, Boundary RG flow associated with the AKNS soliton hierarchy, J. Phys. A 39 (2006) 12889-12926.

[12] F.A. Smirnov, Baxter equations and deformation of Abelian differentials, in: Proceedings of 6th International Workshop on Conformal Field Theory and Integrable Models, Int. J. Mod. Phys. A 19 (2004) 396-417.

[13] A. Klümper, P.A. Pearce, Finite-size corrections and scaling dimensions of solvable lattice models: An analytic method, Phys. Rev. Lett. 66 (1991) 974-977.

[14] A. Fring, C. Korff, B.J. Schulz, The ultraviolet behavior of integrable field theories, affine Toda field theory, Nucl. Phys. B 549 (1999) 579-612.

[15] V.E. Korepin, N.M. Bogoliubov, A.G. Izergin, Quantum Inverse Scattering Method and Correlation Functions, Cambridge Univ. Press, 1993.

[16] C.N. Yang, C.P. Yang, J. Math. Phys. 10 (1969) 122.

[17] A.B. Zamolodchikov, Al.B. Zamolodchikov, Structure constants and conformal bootstrap in Liouville field theory, Nucl. Phys. B 477 (1996) 577-605.

[18] J. Teschner, in preparation.

[19] V.V. Bazhanov, S.L. Lukyanov, A.B. Zamolodchikov, Integrable structure of conformal field theory, I, Commun. Math. Phys. 177 (1996) 381-398;

V.V. Bazhanov, S.L. Lukyanov, A.B. Zamolodchikov, Integrable structure of conformal field theory, II, Commun. Math. Phys. 190 (1997) 247-278;

V.V. Bazhanov, S.L. Lukyanov, A.B. Zamolodchikov, Integrable structure of conformal field theory, III, Commun. Math. Phys. 200 (1999) 297-324. 
[20] P. Dorey, R. Tateo, Anharmonic oscillators, the thermodynamic Bethe ansatz, and nonlinear integral equations, J. Phys. A 32 (1999) L419-L425.

[21] V.V. Bazhanov, S.L. Lukyanov, A.B. Zamolodchikov, Spectral determinants for Schroedinger equation and Q-operators of conformal field theory, J. Stat. Phys. 102 (2001) 567-576.

[22] J. Teschner, A lecture on the Liouville vertex operators, Int. J. Mod. Phys. A 19 (2) (2004) 436-458.

[23] J. Teschner, Liouville theory revisited, Class. Quantum Grav. 18 (2001) R153-R222.

[24] H. Dorn, H.-J. Otto, On correlation functions for non-critical strings with $c<1$ but $d>1$, Phys. Lett. B 291 (1992) 39-43;

H. Dorn, H.-J. Otto, Two and three-point functions in Liouville theory, Nucl. Phys. B 429 (1994) 375-388.

[25] C. Destri, H.J. de Vega, New approach to thermal Bethe ansatz, Phys. Rev. Lett. 69 (1992) 2313.

[26] C. Destri, H.J. de Vega, Unified approach to thermodynamic Bethe ansatz and finite size corrections for lattice models and field theories, Nucl. Phys. B 438 (1995) 413.

[27] C. Destri, H.J. de Vega, Non-linear integral equation and excited-states scaling functions in the sine-Gordon model, Nucl. Phys. B 503 (1997) 621.

[28] G. Feverati, F. Ravanini, G. Takacs, Nonlinear integral equation and finite volume spectrum of sine-Gordon theory, Nucl. Phys. B 540 (1999) 543-586.

[29] V.V. Bazhanov, S.L. Lukyanov, A.B. Zamolodchikov, Integrable quantum field theories in finite volume: Excited state energies, Nucl. Phys. B 489 (1997) 487-531. 